\title{
CCD and HI observations of LSB dwarf galaxies in the general field
}

\author{
V.E. Karachentseva ${ }^{1}$, P. Prugniel ${ }^{2}$, J. Vennik ${ }^{3}$, G.M. Richter ${ }^{4}$, T.X. Thuan ${ }^{5}$ and J.M. Martin ${ }^{6}$ \\ 1 Astronomical Observatory of Kiev University, Kiev, Ukraine \\ 2 Observatoire de Haute Provence, France \\ 3 Tartu Observatory, EE2444 Tartu, Estonia \\ 4 Astrophysical Institute Potsdam, An der Sternwarte 16, D-14482 Potsdam- Babelsberg, Germany \\ 5 Astronomy Department, University of Virginia, Charlottesville,VA 22903, U.S.A. \\ 6 ARPEGES, Place Jules Janssen, F-92195, Meudon Cedex, France
}

Received July 26; accepted November 21, 1995

\begin{abstract}
CCD $B$ and $V$ observations of 16 low-surface-brightness galaxies and HI data for a subsample of 4 of them are presented. Both paired and isolated dwarf galaxies are observed to study possible environmental effects on their characteristics. Most of the observed galaxies are true dwarfs with $-8.0 \geq M_{B} \geq-16.0$, blue colours $(0.16 \leq B-V \leq 0.76)$ and low central surface brightnesses $22.2 \leq \mu_{B} \leq 24.0 \mathrm{mag} \operatorname{arcsec}^{-2}$. Most of them show exponential light profiles with a central light depression. About half of them have bright generally blue knots or peculiar loops.
\end{abstract}

Key words: dwarf galaxies: surface photometry, HI observations

\section{Introduction}

Low surface brightness dwarf galaxies (LSBDGs) are well recognized laboratories in which to study problems of galaxy formation and evolution. They can also be used as test particles for evaluating the possible environmental influences on the general characteristics of dwarfs such as morphological type, linear diameter and luminosity function, colours, mass-to-luminosity ratios, etc.

This influence can be "global", i.e. on the scale of galaxy clusters, "local", i.e. on the scale of groups and cluster subgroups, or in extreme cases due to the "point influence" of a luminous and massive parent galaxy on its dwarf satellite.

An example of global environmental influence is the well-established morphological segregation of LSBDGs in the Local Supercluster: gas-poor (elliptical) dwarfs are predominantly concentrated in rich clusters like Virgo and Fornax, and gas-rich (irregular) dwarfs are loosely distributed in groups and the general field. In other words, the gas content of the LSBDGs correlates with the density of their environment.

Such a specific distribution of gas-poor dwarf galaxies permits us to study their characteristics without the knowledge of their radial velocities, because they are most probably members of the corresponding rich aggregates of galaxies.

Send offprint requests to: G.M. Richter
In the Virgo cluster gas-poor and gas-rich dwarfs show different distributions and their luminosity functions are different (Binggeli et al. 1985). However, the diameters and luminosities of LSBDGs of the same morphology are correlated neither with their Virgocentric distances (Reaves 1983) nor with the local galaxy density (Karachentseva \& Vavilova 1995). The environmental influences on the galaxy luminosity function are considered in the review paper of Binggeli et al. (1988).

The situation for gas-rich dwarf galaxies is quite different from that of gas-poor ones. In order to obtain absolute characteristics of gas-rich dwarfs one needs to determine their radial velocities. During the two last decades, latetype LSBDGs have been intensively studied both in large HI-surveys (Fisher \& Tully 1975; Thuan \& Seitzer 1979; Fisher \& Tully 1981; Schneider et al. 1990; Schneider et al. 1992) and in selected small aggregates.

The question about possible environmental influences on the dwarf population in groups is still much debated (see, for example, Côté et al. 1994; Huchtmeier \& Skillman 1994). Several interesting searches for bound dwarf satellites around bright galaxies have been carried out during the last few years. Results of these studies are discussed by Zaritsky (1994).

Our observing program was motivated by the results of the cluster analysis (CA) studies carried out on a combined sample of LSBDGs from the Catalogue of Karachentseva \& Sharina (1988), (hereafter KS), and the 
Table 1. General characteristics of observed galaxies

\begin{tabular}{|c|c|c|c|c|c|c|c|c|c|c|}
\hline Ident. & $\alpha_{1950}$ & $\delta_{1950}$ & $\begin{array}{c}a \\
\left({ }^{\prime}\right)\end{array}$ & $b / a$ & mag & $\begin{array}{c}V_{\mathrm{h}} \\
(\mathrm{km} / \mathrm{s})\end{array}$ & Source & Type & $\begin{array}{l}\text { Clustering } \\
\text { properties }\end{array}$ & Frames \\
\hline (1) & $(2)$ & (3) & (4) & (5) & (6) & $(7)$ & (8) & (9) & $(10)$ & (11) \\
\hline LGS 3 & $01^{h} 01^{m} 2$ & $21^{\circ} 37^{\prime}$ & 2.2 & 1.00 & 15.4 & -281 & RC3 & $\mathrm{dIm}$ & LG? & $2 \mathrm{~B}, 1 \mathrm{~V}$ \\
\hline U $\quad 672$ & 0101.3 & 4441 & 1.4 & 0.64 & 15.2 & 708 & STMM & dIr & isol. & $1 \mathrm{~B}, 1 \mathrm{~V}$ \\
\hline U 2684 & 0317.6 & 1707 & 2.0 & 0.50 & 15.2 & 351 & $\mathrm{TS}$ & $\mathrm{dIr} / \mathrm{dS}$ & isol. & $2 \mathrm{~B}, 2 \mathrm{~V}$ \\
\hline K 35 & 0322.4 & -0313 & 0.5 & 0.50 & & & & $\mathrm{dIr}$ & isol. & $1 \mathrm{~B}, 1 \mathrm{~V}$ \\
\hline Camel & 0419.4 & 7242 & 4.5 & 0.75 & & & & $\mathrm{dSph} / \mathrm{dIr}$ & pw N1560? & $3 \mathrm{~B}, 1 \mathrm{~V}$ \\
\hline UA 92 & 0427.4 & 6330 & 2.0 & 0.50 & 13.8 & -99 & RC3 & $\mathrm{dIm}$ & pw N1569 & $1 \mathrm{~B}, 1 \mathrm{~V}$ \\
\hline K 47 & 0449.8 & 2306 & 1.0 & 0.80 & 14.8 & & & $\mathrm{dIm}$ & isol. & $1 \mathrm{~B}, 1 \mathrm{~V}$ \\
\hline U 3698 & 0705.7 & 4428 & 1.2 & 0.58 & 16.3 & 420 & STMM & $\mathrm{dIm}$ & pw N2337 & $1 \mathrm{~B}, 1 \mathrm{~V}^{*}$ \\
\hline U 4466 & 0830.3 & 7800 & 0.8 & 1.00 & 15.0 & 1424 & STMM & $\mathrm{dS}$ & pw N2591 & $1 \mathrm{~B}, 1 \mathrm{~V}^{*}$ \\
\hline An 0849 & 0849.1 & 7827 & 1.1 & 1.00 & & & & dSph & pw N2655 & 1B \\
\hline U 4683 & 0854.0 & 5917 & 1.3 & 0.62 & 14.8 & 928 & STMM & $\mathrm{dIr}$ & pw N2685 & $1 \mathrm{~B}, 1 \mathrm{~V}^{*}$ \\
\hline U 6016 & 1051.2 & 5434 & 2.2 & 0.73 & 15.2 & 1493 & RC3 & $\mathrm{dS}$ & pw N3448 & $1 \mathrm{~B}, 1 \mathrm{~V}^{*}$ \\
\hline K 81 & 1152.6 & 4424 & 0.6 & 0.83 & & & & $\mathrm{dIr}$ & pw N3938 & $1 \mathrm{~B}, 1 \mathrm{~V}^{*}$ \\
\hline U 11392 & 1900.5 & 3443 & 1.0 & 0.20 & 17.7 & & & dS & isol. & $1 \mathrm{~B}, 1 \mathrm{~V}$ \\
\hline U 12771 & 2343.0 & 1658 & 1.1 & 0.54 & 16.1 & 1291 & STMW & $\mathrm{dS}$ & isol. & $1 \mathrm{~B}, 1 \mathrm{~V}$ \\
\hline U 12894 & 2357.8 & 3213 & 1.0 & 1.00 & 17.0 & 335 & STMM & $\mathrm{dIm}$ & isol. & $3 \mathrm{~B}, 2 \mathrm{~V}$ \\
\hline
\end{tabular}

Cols.(1)-(5): The data are taken from KS (Karachentseva \& Sharina 1988).

Col.(6): Magnitudes from PGC (Paturel et al. 1989).

Col.(8): $V_{\mathrm{h}}$ sources are: RC3 (de Vaucouleurs et al. 1991); STMM (Schneider et al. 1992); TS (Thuan \& Seitzer 1979); STMW (Schneider et al. 1990).

Col.(9): Type by our estimation.

Col.(10): The clustering properties from our cluster analysis.

Col.(11): The asterisk denotes the exposure time: $30^{\mathrm{min}}$ in $B$ and $20^{\mathrm{min}}$ in $V$.

galaxies with $V_{\mathrm{h}}<2000 \mathrm{~km} / \mathrm{s}$ from the Catalogue of Principal Galaxies (Paturel et al. 1989), (hereafter PGC). Excluding the regions of the Virgo and Fornax clusters and performing the cluster analysis with different clustering radii for this sample of field galaxies, we obtained a set of groups, pairs and isolated galaxies from which various subsamples have been chosen (Karachentseva \& Vavilova 1994a, b).

Actually we selected a subsample of about 100 pairs, which consist of one LSBDG and one normal galaxy, with a projected separation not exceeding $150 \mathrm{kpc}$. Another sample of 150 isolated LSBDGs, having no neighbours within a projected separation of 2 degrees, was selected for comparison.

Our interest in this program is twofold. The first aim is to obtain accurate photometric and HI data for LSBDGs and calculate their intrinsic characteristics and mass-toluminosity ratios. The second goal is to compare the characteristics of paired dwarfs to those of isolated dwarfs in order to search for possible environmental influences.

In this article we present the photometric data for 16 LSBDGs and HI observations for a subsample of 4 LSBDGs. The astrophysical analysis, together with other new data, follows in another paper. A Hubble parameter of $H_{0}=75 \mathrm{~km} / \mathrm{s} / \mathrm{Mpc}$ is used throughout the paper.

\section{Observations}

\subsection{CCD observations}

In two observing runs, during September 1993 and February 1994, we used the Tektronix $512 \times 512$ CCD camera at the Observatoire de Haute-Provence $1.2 \mathrm{~m}$ telescope. The scale is $0.75 \mathrm{arcsec} /$ pixel and the gain is $6.46 \mathrm{e}^{-} / \mathrm{adu}$.

Due to very unstable weather conditions (clouds, cirrus, wind), we could only use 4 of the allocated 14 nights in the two observing runs. The typical seeing was about $2-3$ arcsec in September and about 5 arcsec in February. We obtained $B$ frames for 16 and $V$ frames for 15 objects with a standard exposure time of $2 \times 900$ seconds. The catalogued data of the observed galaxies and the basic characteristics of the CCD frames are listed in Table 1.

The mean sky brightness during these 4 nights was $22.2 \pm 0.23 \mathrm{mag} / \square^{\prime \prime}$ in $B$, and $21.3 \pm 0.16 \mathrm{mag} / \square^{\prime \prime}$ in $V$.

For the photometric calibration, we observed standard stars from Landolt (1992) and Zickgraf et al. (1990). 


\subsection{HI observations}

The 21-cm observations were carried out in 1994 with the Nancay 300-m radiotelescope, which has a HPBW of $4^{\prime}$ $(\mathrm{EW}) \times 22^{\prime}(\mathrm{NS})$ at the declination of 0 degrees. We used a two-channel dual-polarisation receiver with a system temperature of about $40 \mathrm{~K}$. None of the dwarf galaxies included in the HI program had known velocities. For $\mathrm{K} 35, \mathrm{~K} 47$, and $\mathrm{K} 81$, the 1024 channel autocorrelator was operated in a velocity-search mode in which 4 correlator segments, each with 256 channels and a bandwidth of $6.4 \mathrm{MHz}$, were overlapped (typically by $0.7 \mathrm{MHz}$ ) to cover a total velocity search range from 0 to $4800 \mathrm{~km} / \mathrm{s}$, with a channel spacing of $5.28 \mathrm{~km} / \mathrm{s}$ before smoothing. For Camel, suspected to be a nearby galaxy because of its resolution in stars, we searched only in the velocity range -600 to $1600 \mathrm{~km} / \mathrm{s}$.

The observations were made in the standard totalpower (position switching) mode with 2 minute onsource/2 minute off-source integrations. Typically 1 hour was spent on the galaxy and 1 hour on the comparison field, leading to a typical rms noise per channel of $2.5 \mathrm{mJy}$, when smoothing the channel spacing to $21 \mathrm{~km} / \mathrm{s}$.

\section{Reduction and calibration of the CCD frames}

The bias-subtracted and flat-fielded CCD frames were searched for cosmic ray hits and possible "hot pixels" by looking for pixels above the expected noise and checking if they had a point spread function smaller than the estimated seeing. If found, the faulty pixels were replaced interactively by the median value of the surrounding area. To improve the signal-to-noise ratio, we applied the adaptive filtering technique described in Lorenz et al. (1993). This filter recognizes the local signal resolution and adapts its own impulse response to this resolution. As a result the adaptive (space variable) filter smoothes extensively the background, less extensively the galaxian outskirts and not at all the highest resolution features. Before applying the adaptive filter, a mask frame was constructed, matching the observed frame, where bright stars and galaxies were masked out. The mask frame is essential for a proper determination of the noise statistics used by the filter. The filter size and the strength of the filter are variable.

Next, the sky background level was determined on the smoothed frame. The images of stars were carefully masked using a polygon editor before the background interpolation. After background subtraction, the galaxy was interactively cleaned from disturbing objects (foreground stars projected on the galaxy etc.) using the polygon editor again.

\section{Surface photometry algorithm}

\subsection{Profile extraction}

The main data resulting from the present study are the surface brightness profiles. We determined the profiles twice. First round, we calculated azimuthally averaged equivalent brightness profiles using the surface photometry routines developed at the Potsdam Astrophysical Institute. In this way we reduce the surface photometry to a one-dimensional photometry. Since in this case, we sum up the pixel intensities over an increasing area, this method yields higher signal-to-noise ratio for the faint outskirts of the galaxies. More precisely, we count the pixel intensities in areas between successive isophotes in predefined narrow magnitude intervals. The differential counts reduced to a unit area give the azimuthally averaged intensity profile of the galaxy as a function of equivalent radius, defined as in the RC2 (de Vaucouleurs et al. 1976), to be the radius of the circle of the same area as that inside the corresponding irregular isophote. The equivalent light profile was used to extract the isophotal radii, mean surface brightnesses and to determine the best-fitting parameters of the particular density distribution models (see Sect. 4.3). In addition to the differential radial surface brightness profiles we calculated the growth curve of the galaxy by summing up the pixel values from the centre outwards in successive isophotes. The total magnitude $\left(B_{T}\right)$ was estimated by asymptotic extrapolation of this radial growth curve.

In order to recover some information about the twodimensional structure such as ellipticities and position angles of isophotes, we used in a second round of calculations the ellipse fitting algorithm, which is based on the formulas given in Bender \& Möllenhoff (1987) in its realization in the SURFPHOT package running within MIDAS. By this method the ellipse fitting procedure is started from the central intensity peak. The center of the innermost ellipse is determined by fitting a gaussian to the subimage in both the $x$ - and $y$-directions. The lower level ellipses are equally spaced in surface brightnesses. The centers of the lower level ellipses as well as their orientation and axis ratios are free parameters, which are recalculated for every narrow surface brightness interval of the studied galaxy. At the end of the fitting process a smooth galaxy model is constructed. The subtraction of this model from the original image allows us to remove the smooth elliptical component and to detect underlying structures (spiral arms, dust, HII regions) if any.

As the result of the ellipse fitting we obtained a set of radial profiles: surface brightness (SB), minor-to-major axis ratio $(b / a)$, position angle $(\mathrm{PA})$ - in both $B$ and $V$ colours. Combining the $B$ and $V$ surface brightness profiles, which were obtained with the one particular set of fitting ellipses applied to both the $B$ and $V$ frames, the colour profile was constructed. 
Table 2. Model-free photometric data

\begin{tabular}{|c|c|c|c|c|c|c|c|c|c|c|c|}
\hline Ident. & $\begin{array}{c}B_{T} \\
(\mathrm{mag})\end{array}$ & $\begin{array}{c}B_{25} \\
(\mathrm{mag})\end{array}$ & $\begin{array}{l}r_{25} \\
\left({ }^{\prime \prime}\right)\end{array}$ & $\begin{array}{l}B_{26.5} \\
(\mathrm{mag})\end{array}$ & $\begin{array}{c}r_{26.5} \\
\left({ }^{\prime \prime}\right)\end{array}$ & $(b / a)_{26.5}$ & $\begin{array}{c}\mathrm{PA}_{26.5} \\
\left({ }^{\circ}\right)\end{array}$ & $\begin{array}{c}\mu_{B, 0} \\
\left(\mathrm{mag} / \square^{\prime \prime}\right)\end{array}$ & $\begin{array}{c}B_{T}-V_{T} \\
(\mathrm{mag})\end{array}$ & $\begin{array}{c}(B-V)_{0} \\
(\mathrm{mag})\end{array}$ & $\begin{array}{c}(B-V)_{\mathrm{ar}} \\
(\mathrm{mag})\end{array}$ \\
\hline$(1)$ & $(2)$ & $(3)$ & (4) & $(5)$ & $(6)$ & $(7)$ & $(8)$ & (9) & (10) & $(11)$ & $(12)$ \\
\hline LGS 3 & 16.18 & & 3.0 & 17.05 & 36. & 0.67 & 175 & 25.4: & 0.73 & 0.55 & 0.68 \\
\hline U $\quad 672$ & 17.03 & 17.75 & 13.4 & 17.12 & 23.2 & 0.63 & 134 & 24.0 & 1.30 & 1.00 & \\
\hline U 2684 & 16.16 & 16.70 & 19. & 16.28 & 30.5 & 0.25 & 107 & 23.55 & 0.46 & 0.50 & 0.44 \\
\hline K 35 & 17.88 & 18.27 & 9.8 & 17.93 & 14.1 & 0.60 & 146 & 23.75 & 0.73 & 1.05 & 0.74 \\
\hline Camel & 14.84 & 18.10 & 12.5 & 15.10 & 78. & 0.57 & 69 & 24.2 & 0.83 & 1.00 & 0.81 \\
\hline UA 92 & 15.22 & 18.00 & 13. & 15.75 & 58. & 0.45 & 73 & 25.1 & 1.34 & 1.20 & 1.29 \\
\hline K 47 & 16.40 & 16.97 & 19. & 16.70 & 26.6 & 0.67 & 15 & 23.2 & 0.88 & 0.70 & 0.91 \\
\hline U 3698 & 15.41 & 15.63 & 24. & 15.45 & 34.5 & 0.72 & 10 & 22.7 & 0.47 & 0.50 & 0.46 \\
\hline $\mathrm{U} \quad 4466$ & 16.00 & 16.25 & 21. & 16.05 & 29. & 0.68 & 40 & 23.15 & 0.46 & 0.56 & 0.44 \\
\hline An 0849 & 17.60 & & 3.5 & 17.72 & 22 . & 0.95 & 20 & 24.5 & & & \\
\hline U 4683 & 15.81 & 16.30 & 22.5 & 15.85 & 35.3 & 0.63 & 108 & 23.45 & 0.50 & 0.50 & 0.48 \\
\hline U 6016 & 14.88 & 15.35 & 32.5 & 14.95 & 54. & 0.78 & 63 & 22.4 & 0.21 & 0.35 & 0.34 \\
\hline K 81 & 16.10 & 16.60 & 20.4 & 16.20 & 32. & 0.90 & 64 & 23.7 & 0.50 & 0.55 & \\
\hline U 11392 & 15.87 & 16.20 & 17. & 15.97 & 28. & 0.58 & 107 & 22.0 & 0.40 & 0.65 & 0.59 \\
\hline U 12771 & 16.45 & 16.80 & 16.6 & 16.54 & 24.7 & 0.47 & 121 & 23.2 & 0.83 & 0.70 & 0.77 \\
\hline U 12894 & 16.36 & 17.05 & 17.6 & 16.55 & 30. & 0.86 & 114 & 24.4 & 0.37 & 0.40 & 0.38 \\
\hline
\end{tabular}

There is a good agreement between the radial SB profiles, calculated using the Potsdam surface photometry routines and the ellipse fitting algorithm implemented in MIDAS. That means, the obtained SB profiles are probably not very sensitive to the surface photometry methods applied. The whole set of radial profiles is given for each galaxy in Figs. 3-18. Because of the smearing due to seeing effects, the brightness distribution is flattened near the center. Therefore, the isophotes are rounder and the position angles are uncertain. According to Franx et al. (1989) the seeing can affect these parameters at radii as large as about 5 times the seeing's full-width at halfmaximum (FWHM). In the periphery, the radial profiles can be reasonably extended to the $\mu_{B} \sim 27.5 \mathrm{mag} / \square^{\prime \prime}$ isophote. At fainter levels the profiles are severely affected by sky background uncertainties.

Three different types of colours were determined.

i) central colour: determined by means of extrapolation of the colour profile to the center.

ii) luminosity weighted colour: determined, in our case, as the difference of the asymptotic $B$ and $V$ magnitudes, and dominated by the colours of the bright parts of the galaxy.

iii) area weighted colour: determined as the average of the colours of the intervals between the ellipses in the differential colour profile.

The area weighted colour should be more representative for the underlying colour of the disk population, while the luminosity weighted colour is appropriate for comparison with colours derived using aperture photometry. In our case the luminosity and area weighting procedures yield generally similar results, since the bulge is usually weak in the studied LSB galaxies.

The observed photometric data are summarized in Table 2, where the data are arranged as follows:

Column 1: UGC (Nilson 1973) name (U) or the galaxy designation according to Karachentseva \& Sharina (1988). Column 2: Asymptotic B-magnitude.

Column 3: Integrated magnitude within $25^{\text {th }}$ magnitude isophote.

Column 4: Galaxy equivalent radius (in seconds of arc) determined at the $25^{\text {th }}$ magnitude isophote.

Column 5: Integrated magnitude within the $26.5^{\text {th }}$ magnitude Holmberg isophote.

Columns 6-8: Galaxy equivalent radius $r_{26.5}$, minor-tomajor axis ratio $(b / a)_{26.5}$ and position angle measured counterclockwise from North $\mathrm{PA}_{26.5}$ (in degrees) of the fitted ellipses, determined at the $26.5^{\text {th }}$ magnitude isophote. Column 9: Surface brightness of the galaxy extrapolated to the center.

Columns 10-12: Luminosity weighted colour $B_{T}-V_{T}$, central colour $(B-V)_{0}$ and area weighted colour $(B-V)_{\text {ar }}$, obtained by averaging over the radial colour index profile.

The photometric parameters, reduced for galaxy distance and for absorption within our Galaxy, are listed in Table 3, where the data are arranged as follows:

Column 1: Galaxy name.

Column 2: Galaxy distance (in Megaparsecs) determined for the majority of the galaxies from measured redshifts corrected to the local rest frame using the IAU convention $V_{0}=V_{\mathrm{h}}+300 \sin l \cos b(\mathrm{~km} / \mathrm{s})$. For LGS 3 and UA 92 we used the photometric distance moduli estimates. For An 
0949+78 the distance of NGC 2655 was assigned (see the description of objects).

Column 3: Central surface brightness, corrected for absorption within our Galaxy, according to $A_{\mathrm{b}}=$ $-0.2 \operatorname{cosec} b^{\mathrm{II}}$.

Column 4: Galaxy equivalent radius (in kiloparsecs), determined at the $26.5^{\text {th }}$ magnitude isophote.

Column 5: Absolute B-magnitude, corrected for absorption within our Galaxy.

Columns 6, 7: Central colour $(B-V)_{0}^{\mathrm{c}}$ and luminosity weighted colour $\left(B_{T}-V_{T}\right)^{\mathrm{c}}$, both corrected for reddening $E(B-V)$ within the Galaxy, calculated from the relation $A_{\mathrm{b}}=4 E(B-V)$.

For Camel, UGCA 92 and UGC 12771 the corrections for absorption within our Galaxy are uncertain due to their location near to the galactic plane. Uncertain measurements are denoted with a colon.

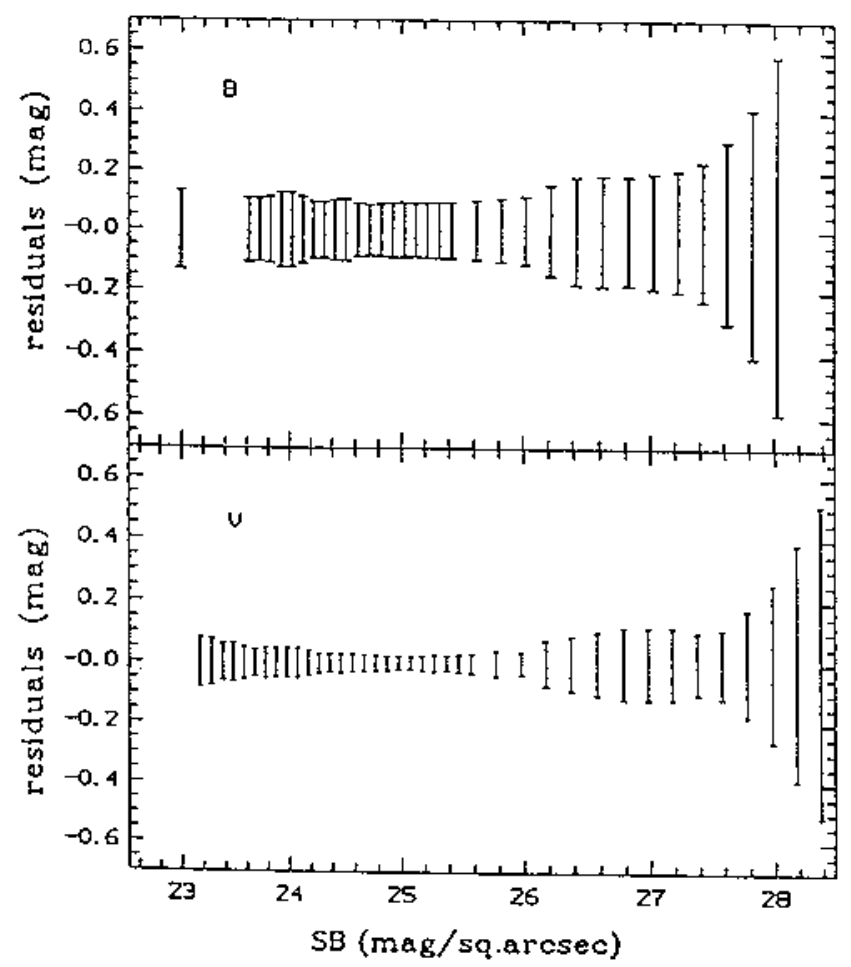

Fig. 1. Internal accuracy of surface brightness profiles: the $1 \sigma$ deviations of surface brightnesses of individual profiles from their mean values are plotted as error bars for $10 \mathrm{~B}$ frames (top panel) and for $4 \mathrm{~V}$ frames (bottom panel) as a function of surface brightness (see text)

\subsection{The surface photometry errors}

The magnitude errors consist of internal and external components. The internal component is dominated by the error in the adopted mean sky value. As measured on the filtered CCD frames, it is typically $0.4 \%$ for the $V$ frames and $0.6 \%$ for the $B$ frames. Because the typical sky surface brightness is between 21.7 and $22.3 \mathrm{Bmag} / \square^{\prime \prime}$, our surface brightness profiles are limited to $\mu_{B} \leq 27.5 \mathrm{mag} / \square^{\prime \prime}$, beyond which the error in the mean sky begins to exceed the signal from object. To check the internal consistency of our photometric profiles we have intercompared individual profiles for the same object obtained from different frames in the same passband. As indicated in Table 1 we have two $B$ frames each for LGS 3 and UGC 2684 and three $B$ frames each for Camel and UGC 12894. All these frames were processed individually and the residuals between every single profile and the mean profile were calculated for each galaxy.

The $1 \sigma$ dispersion of these residuals is plotted as error bars in Fig 1 (top panel). The mean error is roughly constant at $\sigma \approx 0.11 \mathrm{Bmag} / \square^{\prime \prime}$ out to the Holmberg isophote of $\mu_{B}=26.5 \mathrm{mag} / \square^{\prime \prime}$, followed by a rapid increase at lower surface brightnesses. A similar intercomparison was performed for the two $V$ frames of UGC 2684 and UGC 12894 . The resulting $1 \sigma$ error bars are given in Fig. 1 (bottom panel). Despite very poor statistics, it is obvious, that the consistency of the $V$ profiles is better than that of the $B$ profiles and there is a mean error of about $0.07 \mathrm{mag} / \square^{\prime \prime}$ out to the $\mu_{V} \sim 26.0 \mathrm{mag} / \square^{\prime \prime}$ isophote. We estimate the total magnitude internal errors to be less than $0.2 \mathrm{mag}$ in $B$ and $0.15 \mathrm{mag}$ in $V$.

Unfortunately, there are no reliable photometric data available to evaluate our true external errors. The comparison of our blue total magnitudes with those given in the PGC for 12 galaxies in common, yields a poor agreement: $B_{T \text {.our }}-m_{\mathrm{PGC}}=0.44 \pm 1.08 \mathrm{mag}$, with a large scatter. The comparison of our data with those obtained by Staveley-Smith et al. (1992), for two common galaxies shows instead a good agreement (see the description of individual objects in Sect. 5).

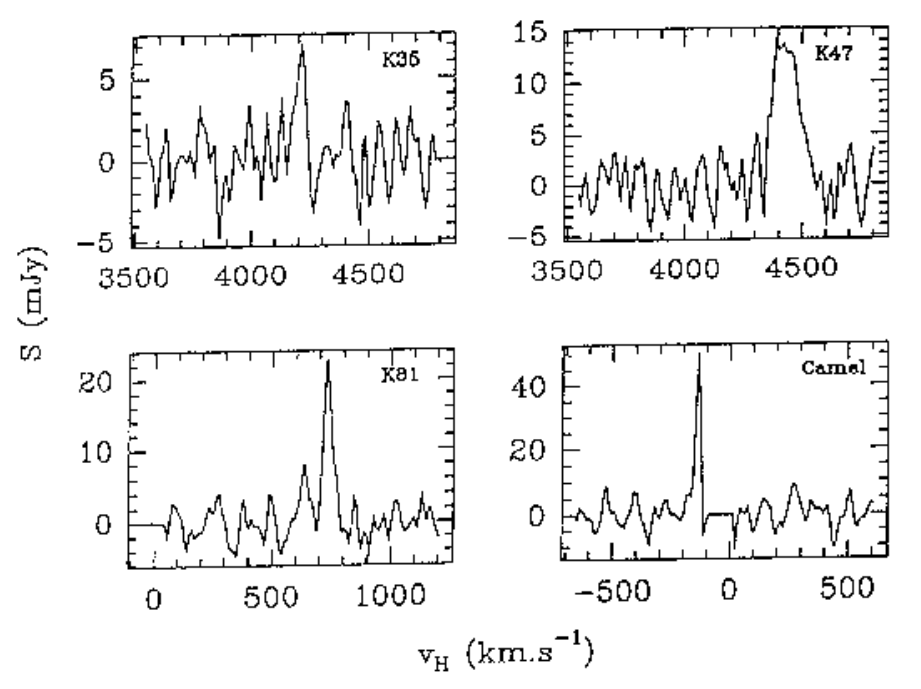

Fig. 2. The HI profiles for the 3 detected dwarf galaxies and the $\mathrm{HI}$ spectrum around $0 \mathrm{~km} / \mathrm{s}$ for Camel 
Table 3. Derived photometric parameters

\begin{tabular}{lcccccc}
\hline \hline Ident. & $\begin{array}{c}\text { Dist. } \\
(\mathrm{Mpc})\end{array}$ & $\begin{array}{c}\mu_{B, 0}^{\mathrm{c}} \\
(\mathrm{mag} / \square ”)\end{array}$ & $\begin{array}{c}r_{26.5} \\
(\mathrm{kpc})\end{array}$ & $\begin{array}{c}M_{B}^{\mathrm{c}} \\
(\mathrm{mag})\end{array}$ & $\begin{array}{c}(B-V)_{0}^{\mathrm{c}} \\
(\mathrm{mag})\end{array}$ & $\begin{array}{c}\left(B_{T}-V_{T}\right)^{\mathrm{c}} \\
(\mathrm{mag})\end{array}$ \\
\hline$(1)$ & $(2)$ & $(3)$ & $(4)$ & $(5)$ & $(6)$ & $(7)$ \\
\hline & & & & & & \\
LGS 3 & 0.58 & $25.1:$ & 0.10 & -7.94 & 0.47 & 0.65 \\
U 672 & 12.52 & 23.4 & 1.41 & -14.11 & 0.84 & 1.14 \\
U 2684 & 5.45 & 23.2 & 0.81 & -12.89 & 0.40 & 0.36 \\
K 35 & 52.84 & 23.5 & 3.62 & -16.01 & 0.98 & 0.66 \\
Camel & & 23.5 & & & 0.80 & 0.63 \\
UA 92 & 2.21 & $24.2:$ & 0.62 & $-12.6:$ & $0.9:$ & $1.1:$ \\
K 47 & 59.29 & $22.3:$ & 7.6 & $-18.3:$ & $0.4:$ & $0.6:$ \\
U 3698 & 6.05 & 22.2 & 1.01 & -14.03 & 0.37 & 0.34 \\
U 4466 & 21.35 & 22.8 & 3.00 & -16.03 & 0.47 & 0.37 \\
An 0849 & 21.16 & 24.1 & 2.26 & -14.41 & & \\
U 4683 & 13.59 & 23.1 & 2.33 & -15.18 & 0.42 & 0.42 \\
U 6016 & 20.95 & 22.2 & 5.48 & -16.97 & 0.30 & 0.16 \\
K 81 & 10.45 & 23.5 & 1.62 & -14.20 & 0.50 & 0.45 \\
U 11392 & & $21.1:$ & & & $0.4:$ & $0.2:$ \\
U 12771 & 20.08 & 22.9 & 2.40 & -15.35 & 0.63 & 0.76 \\
U 12894 & 7.89 & 23.8 & 1.15 & -13.66 & 0.27 & 0.24 \\
\hline \hline
\end{tabular}

Table 4. Results of the model-fitting of light profiles

\begin{tabular}{lcccccccr}
\hline \hline Ident. & $n$ & $A$ & $B$ & $\begin{array}{c}r_{\text {ef }}^{*} \\
\left({ }^{\prime \prime}\right)\end{array}$ & $\begin{array}{c}\mu_{\text {ef }}^{*} \\
\left(\mathrm{Bmag} / \square^{\prime \prime}\right)\end{array}$ & $\begin{array}{c}\sigma_{\text {fit }} \\
(\mathrm{mag})\end{array}$ & $\begin{array}{c}\mu_{\text {start }} \\
\left(\mathrm{Bmag} / \square^{\prime \prime}\right)\end{array}$ \\
\hline$(1)$ & $(2)$ & $(3)$ & $(4)$ & $(5)$ & $(6)$ & $(7)$ & $(8)$ & $(9)$ \\
\hline & & & & & & & & \\
LGS 3 & 0.7 & 25.69 & 0.0048 & 47 & 26.86 & 0.018 & 25.8 & 27.5 \\
U 672 & 0.5 & 24.21 & 0.0043 & 13.1 & 24.94 & 0.063 & 24.3 & 27.5 \\
U 2684 & 0.8 & 23.16 & 0.0470 & 14.9 & 24.54 & 0.198 & 23.7 & 28.0 \\
K 35 & 0.4 & 24.03 & 0.0033 & 5.2 & 24.24 & 0.086 & 23.8 & 27.0 \\
Camel & 1.0 & 24.76 & 0.0215 & 84 & 26.57 & 0.021 & 24.8 & 26.5 \\
UA 92 & 1.0 & 24.63 & 0.0306 & 59 & 26.50 & 0.073 & 24.8 & 27.6 \\
K 47 & 0.5 & 23.77 & 0.0038 & 13.9 & 24.50 & 0.075 & 23.9 & 27.5 \\
U 3698 & 1.0 & 21.69 & 0.1378 & 13.2 & 23.51 & 0.020 & 23.3 & 26.5 \\
U 4466 & 0.5 & 23.37 & 0.0038 & 13.9 & 24.10 & 0.047 & 23.2 & 28.0 \\
An 0849 & 0.6 & 24.93 & 0.0086 & 16.8 & 25.88 & 0.019 & 25.0 & 26.5 \\
U 4683 & 0.7 & 23.50 & 0.0185 & 18.1 & 24.66 & 0.026 & 23.5 & 26.5 \\
U 6016 & 1.3 & 22.29 & 0.1923 & 27.6 & 24.76 & 0.064 & 22.5 & 26.8 \\
K 81 & 0.6 & 23.23 & 0.0086 & 16.8 & 24.18 & 0.021 & 23.6 & 28.0 \\
U 11392 & 0.9 & 22.01 & 0.1211 & 10.2 & 23.61 & 0.086 & 22.0 & 26.0 \\
U 12771 & 0.6 & 23.36 & 0.0150 & 12.0 & 24.31 & 0.042 & 23.2 & 27.0 \\
U 12894 & 0.6 & 24.36 & 0.0072 & 18.7 & 25.31 & 0.067 & 24.4 & 27.7 \\
\hline \hline
\end{tabular}

\subsection{Profile fitting and model parameters}

The most commonly used model profile for fitting the brightness distribution of spiral and irregular galaxies is the exponential intensity law

$$
I(r)=I_{0} \mathrm{e}^{-\alpha r}
$$

which has two free parameters: the central surface brightness $\left(I_{0}\right)$ and the exponential scale length $(1 / \alpha)$. De
Vaucouleurs (1948) introduced the $r^{1 / 4}$ law to represent the light profiles of ellipticals and of spheroidal components of disk galaxies:

$$
I(r)=I_{\mathrm{e}} \exp \left(-k\left[\left(\frac{r}{r_{\mathrm{e}}}\right)^{1 / 4}-1\right]\right) .
$$

Sersic (1968) proposed a generalization of the de Vaucouleurs formula, i.e. a power law in which the 
exponent $1 / n$ is a free parameter:

$$
I(r)=I_{0} \operatorname{dex}\left(-r^{1 / n}\right) .
$$

In our earlier study (Vennik \& Richter 1994) we found that the light profiles of low surface brightness dwarf galaxies are predominantly "subexponential" (i.e. show convex curvature). In their recent paper Vader \& Chaboyer (1994) show that radial surface brightness profiles of dwarf galaxies, whether of early or late type, display the same varieties in shape as those of giant galaxies. Only a few are well described by a pure $r^{1 / 4}$ law; exponential profiles prevail, but often show a central depression. Rönnback \& Bergvall (1994) found that about $24 \%$ of blue LSB galaxies which they studied photometrically have a real central light depression.

Thus we need a third parameter, besides the usual two scale parameters, to be able to match the different curvatures of the light profiles of dwarfs. For this reason, we decided to apply the $r^{1 / n}$ model. As demonstrated by Caon et al. (1993) the introduction of a third parameter is not purely a mathematical trick, but has some physical meaning, because $n$ is correlated with global parameters of, at least, early type galaxies. For fitting purposes, Caon et al. (1993) suggested the relation

$$
\mu(r)=A+B r^{1 / n}
$$

where the effective parameters of the $r^{1 / n}$ model are

$$
\begin{gathered}
\mu_{\mathrm{ef}}^{*}=A+c_{n}, \\
r_{\mathrm{ef}}^{*}=\left(c_{n} / B\right)^{n}, \\
c_{n}=2.5(0.868 n-0.142) .
\end{gathered}
$$

For $n=1$ this model coincides with the exponential model, where $B=1.086 \alpha$.

We fitted our equivalent light profiles with relation (4) and determined the best-fitting value of the parameter $n$. In practice, for each galaxy profile we constructed a grid of models with $0.2 \leq n \leq 16$ and computed the rms scatter of the observed-calculated residuals. We then chose as the best value of the exponent the one giving the smallest rms scatter. The radial range of the light profiles over which the best fit has been computed goes from outside the region dominated by the seeing (typically a few seconds of arc) out to where the uncertainties in the sky subtraction render the light profiles unreliable.

The results of the fitting of the $B$ profiles are summarized in Table 4, where the data are arranged as follows:

Column 1: Galaxy name.

Column 2: Parameter $n$ of the best-fitting model for the equivalent $B$-profile.

Columns 3, 4: Coefficients $A$ and $B$ of the fitting formula (4).

Columns 5, 6: Scale parameters $r_{\mathrm{ef}}^{*}$ and $\mu_{\mathrm{ef}}^{*}$ of the model, as defined in (5-7).

Column \%: Rms error of the fit $\sigma_{\text {fit }}$.
Columns 8, 9: Limits $\mu_{\text {start }}$ and $\mu_{\text {end }}$ of the surface brightness range of the fit.

The same model is also appropriate, in most cases, for the $V$ profile, due to a lack of colour gradients in the majority of the sampled galaxies.

The light profiles show typically a central intensity depression (which corresponds to the model with $n<1$ ). Only two galaxies, Camel and UGCA 92, seem to be pure exponentials; UGC 3698 also shows the presence of a outer exponential component. The profile of the brightest galaxy in our sample, UGC 6016, shows a marginal concave curvature which may be indicative of the presence of a small central bulge or nucleus.

\section{Results}

The photometric parameters are summarized in Tables 24. The HI parameters for 3 detected galaxies are given in Table 5 , in which the velocities $v_{50}$ (Col. 2), velocity widths $W_{50}$ and $W_{20}$ (Cols. 3 and 4 ) as well as fluxes (Col. 5) are listed with their errors, calculated following Schneider et al. (1990). No errors were given for K 35 because of its marginal detection.

Table 5. HI parameters of detected dwarf galaxies

\begin{tabular}{lcccc}
\hline \hline Name & $\begin{array}{c}v_{50} \\
(\mathrm{~km} / \mathrm{s})\end{array}$ & $\begin{array}{c}W_{50} \\
(\mathrm{~km} / \mathrm{s})\end{array}$ & $\begin{array}{c}W_{20} \\
(\mathrm{~km} / \mathrm{s})\end{array}$ & $\begin{array}{c}\text { Flux } \\
(\mathrm{Jy} \mathrm{km} / \mathrm{s})\end{array}$ \\
\hline & & & & \\
K 35 & 4205 & 63 & & 0.4 \\
K 47 & $4435 \pm 14$ & $137 \pm 27$ & $190 \pm 42$ & $2.0 \pm 0.3$ \\
K 81 & $735 \pm 7$ & $42 \pm 11$ & $74 \pm 18$ & $1.12 \pm 0.22$ \\
\hline \hline
\end{tabular}

The HI profiles for the 3 detected galaxies and the HI spectrum around $0 \mathrm{~km} / \mathrm{s}$ for Camel are shown in Fig. 2.

The results of the surface photometry are shown in Figs. 3 to 18. On these plots each galaxy is described by three panels: panel A - a 2-dimensional grey scale representation of the surface brightnesses, supplemented by a set of isophotes; panel B - the equivalent $B$ and $V$ surface brightness profiles, derived by means of Potsdam surface photometry routines and the $(B-V)$ colour profile; panel $\mathrm{C}$ - the radial profiles of the parameters of fitted ellipses, which include the axial ratio $(b / a)$, major axis position angle (PA measured counterclockwise from North), and displacements of the center of fitted ellipses both in $x\left(x-x_{0}\right)$ and in $y\left(y-y_{0}\right)$ in seconds of arc, where $\left(x_{0}, y_{0}\right)$ is the center of innermost ellipse.

Since most of studied galaxies have irregular shape, the parameters of the fitted ellipses are subject to large stochastic fluctuations. Thus, the radial profiles on the panel C may look not very informative but they are useful for better understanding and proper interpretation of the $\mathrm{SB}$ and colour profiles given on panel B. 
Next we give a short description of every observed galaxy based on the CCD images, plots of their photometric parameters, and on the available data in the literature.

LGS 3 is one of the lowest surface brightness objects in our sample. It appears partially resolved into stars and knots embedded in a very diffuse and amorphous body. On our frames it appears more elongated than on POSS prints. There is no pronounced nucleus visible. About 6 faint knots are clustered near to the center, and a few further knots are visible in the periphery. We measured the magnitudes and colours of the 7 brightest knots. Three central knots have $B-V$ colours in the range $0.18 \div 0.53$, and peripherial knots are redder $(0.62<B-V<1.11)$. Part of these knots may be projected foreground stars. Central spikes in both light profiles are caused by the bright knots. According to Tikhonov \& Sazonova (1994) the distance of LGS 3 is about $0.58 \mathrm{Mpc}$.

UGC 672 is an amorphous and diffuse dwarf galaxy, without any visible nucleus or bright knots. A star is projected near the center. It has been removed before deriving the profile. Only one 1-minute $V$ frame was obtained for this galaxy yielding poorly determined $V$ and $B-V$ profiles. Despite large uncertainties, the light profiles seem to be non-exponential with a central light depression. According to the results of cluster analysis (hereafter CA) UGC 672 is an isolated dwarf galaxy.

UGC 2684 is a rather bright elongated galaxy with regular isophotes, showing a faint nucleus. Light profiles show a pronounced outer disk component and a central light depression. There is a marginal gradient in the colour profile, $B-V$ getting redder near to the center. According to Thuan \& Seitzer (1979) (TS) and Staveley-Smith et al. (1992) (SDK), the HI profile shows a steep-sided shape, characteristic of a rotating disk. Our magnitude estimate $B_{T}=16.16$ agrees with the multi-aperture photoelectric estimate $B=16.5$, given in SDK. If the HI velocity, given in TS, yields its true distance, then its intrinsic luminosity of about $M_{T}^{\mathrm{c}}=-12.89$, making UGC 2684 a dwarf spiral galaxy. According to the CA criterion, UGC 2684 is an isolated galaxy.

K 35 is a faint small diffuse galaxy, with a very faint reddish nucleus and without any bright knots. It has a clearly non-exponential luminosity profile. According to the CA criterion it should be isolated. In the vicinity of $\mathrm{K} 35$ there is a pair of galaxies NGC 1320/21 with radial velocities 2640 and $2730 \mathrm{~km} / \mathrm{s}$, respectively (Mazzarella \& Balzano 1986). K 35 is marginally detected in HI (see Fig. 2).With a velocity $V_{\mathrm{h}}=4205 \mathrm{~km} / \mathrm{s}, \mathrm{K} 35$ is a background isolated dwarf galaxy with respect to the NGC 1320/21 pair.

Camel was discovered by I.D. Karachentsev in 1991 on the POSS prints. The object is very diffuse, without any clear nucleus but with some bright knots near the center. Many foreground stars are projected onto the galaxy. Due to background subtraction difficulties in the observed frames, our measured radii may be underestimated when compared to the POSS measurements. After removing some bright knots the remaining luminosity profiles are nearly exponential without any colour changes. Camel is partially resolved into stars with the 6-m telescope, but the distance is not yet estimated. With high probability Camel is a companion of the nearby galaxy NGC 1560 (I. Karachentsev, private communication).

For Camel, no feature was detected by HI observations down to a rms level of $2.1 \mathrm{mJy}$ in the $-600 \div+1600$ $\mathrm{km} / \mathrm{s}$ velocity range. There is an emission feature at $v=-132 \mathrm{~km} / \mathrm{s}$ with a peak intensity of $27 \mathrm{mJy}$, which is probably due to a high velocity cloud infalling into the Milky Way.

UGCA 92 is observed as a very low surface brightness amorphous galaxy, partially resolved into stars and knots. There is no visible nucleus, but two loops of knots are placed symmetrically relative to the geometric center of the galaxy. The $B-V$ colours of the two brightest parts of the loops are 0.88 and 1.13 , which are slightly bluer than the colour of the main body of the galaxy. The luminosity profiles are exponential without colour gradient. According to Karachentsev et al. (1994), the distance of UGCA 92 is $2.21 \mathrm{Mpc}$, and it is related to NGC 1569.

K 47 has a very interesting morphology: it has two bright loops (poorly seen on the grey-scale plot in Fig. 9) consisting of many single knots. The southern loop is much brighter, compared to the northern one. They resemble the structures, which we detected in UGCA 92, but in K 47 these loops occupy a larger fraction of the galaxy area. The light profiles consist of two linear parts (two disk components). The inner disk represents the bright loops, the outer disk represents the underlying faint component, which is slightly redder. K 47 is well detected in HI (see Fig. 2). According to the measured redshift and intrinsic parameters it is a distant, probably isolated peculiar galaxy.

UGC 3698 is a dwarf galaxy with regular isophotes and rather bright central part. The light profile is exponential with pronounced central light depression, typical for many dwarf galaxies. Two bright knots have $B-V$ colours of 0.20 and 0.27 , which is slightly bluer than the underlying component. According to the CA criterion NGC 3698 is paired with NGC 2337.

UGC 4466 is a dwarf galaxy of regular shape without any bright knots or other irregularities. It has a round central part but no pronounced nucleus. The luminosity profile is clearly non-exponential showing convex curvature. There is a clear colour gradient, significant at the $17 \sigma$ level, with a reddish center, getting bluer outwards. UGC 4466 is paired with NGC 2591 according to CA criterion.

An $0849+78$ is located at the extension of an outer spiral arm of NGC 2655 and seems to be connected to it by a very faint bridge. Its isophotes are of regular shape; a faint elongated nucleus is marginally visible. The light profile 
is probably non-exponential, but its outer part is poorly determined due to the uncertainties in background subtraction. The $\mathrm{HI}$ observations show that An $0849+78$ is confused by NGC 2655, at $V_{\mathrm{h}}=1400 \mathrm{~km} / \mathrm{s}$. To calculate the absolute characteristics we use for An $0849+78$ the distance of NGC 2655. If An $0849+78$ is a bound (dwarf spheroidal) companion of NGC 2655, its survival in the gravitational field of the bright galaxy is problematic. New multicolour photometry and HI observations are needed.

UGC 4683 is an elongated dwarf galaxy, with some irregularities in its central part: a star (or may be a bright knot) is projected near the center. This star/knot has definitely a redder colour $(B-V=0.98)$ when compared to the colour of the underlying galaxy. The light profile is nearly exponential within 25 arcseconds, showing a steeper gradient in its outer part. According to CA, UGC 4683 is paired with NGC 2685.

UGC 6016 is a disrupted spiral galaxy, with a faint elongated nucleus and a number of bright knots in the periphery. Seven measured knots have colour indices $B-V$ in the range $-0.15 \div 0.29$. The underlying galaxy itself is about as blue as the knots. The light profile is nearly exponential, with some excess light near the center: this is the only galaxy in the sample, which shows a clear positive curvature of its light profile, corresponding to the power law model with $n>1$. The negative colour gradient is caused by the blue knots at the periphery.

UGC 6016 is obviously related to the neighbouring galaxy NGC 3448: a faint jet is directed toward NGC 3448. $\mathbf{K} \mathbf{8 1}$ is an amorphous dwarf galaxy without any resolved nucleus. Its light profile is clearly non-exponentional without a clear colour gradient. Two bright foreground stars, projected on the galaxy, were removed from the galaxy image. K 81 is well detected in HI (Fig. 2). According to CA, K 81 is paired with NGC 3938, but it is probably not a gravitationally bound companion.

UGC 11392 is an elongated galaxy with regular isophotes and a faint nucleus. A foreground star was removed near the center. The light profiles are nearly exponential, without any significant colour changes. This galaxy was not detected in the $-299 \div 6577 \mathrm{~km} / \mathrm{s}$ velocity range by HI observations of Schneider et al. (1990) (STMW). According to CA, UGC 11392 is an isolated galaxy.

UGC $\mathbf{1 2 7 7 1}$ is a dwarf galaxy of regular shape, with a faint nucleus. The surface brightness profile is exponential with a central light depression. The observed colour gradient in the outer parts may be an effect of the incorrect sky subtraction in $B$. The HI line profile shows a "doublehorn" shape (STMW). According to CA, UGC 12771 is an isolated galaxy.

UGC 12894 is an amorphous diffuse dwarf galaxy, without nucleus, but consisting of several bright blue knots, which are concentrated into several "nests". The $B-V$ colours of the 4 brightest knots range from -0.16 to 0.32 .
The surface brightness profile is exponential with a central light depression. The colour gradient is uncertain. The HI line profile is gaussian, with asymmetry (TS, SDK). Our estimate of $B_{T}=16.36$ is in good agreement with $B=16.5$ given by SDK. According to CA, UGC 12894 is an isolated dwarf galaxy.

\section{Summary}

New photometric and HI measurements show that among 16 observed LSB galaxies, 12 of them are intrinsically faint dwarf galaxies with absolute blue magnitudes between -8.0 and -16.0 . Three other galaxies - K 47, UGC 6016 and UGC 11392 - are probably not classical dwarf LSB galaxies. The observed galaxies have blue intrinsic colours $0.2 \leq B_{T}-V_{T} \leq 0.76$ and low central surface brightnesses ranging between 22.2 and $24.0\left(\mathrm{Bmag} / \square^{\prime \prime}\right)$. The red colours of UGC 672 and UGCA 92 are uncertain.

Half of the observed galaxies reveal bright knots or peculiar loops, consisting of many knots, located both near the center and at the periphery. These irregularities are usually bluer than the underlying component, and are probably regions of recent star formation. A faint nucleus is visible in some observed galaxies.

About half of the galaxies show disk components in their light profiles, mostly accompanied by a central light depression. Others are clearly "subexponentials", with a convex curvature when plotted as a function of linear radius, as is most common for dwarf galaxies. Only UGC 6016 shows a concave light profile $(n>1.0)$.

Seven galaxies have close neighbours, others are probably isolated galaxies. The comparison of photometric characteristics of paired and isolated dwarfs does not reveal any statistically significant differences. More detailed analysis with inclusion of additional data will be carried out in a further paper.

Acknowledgements. VEK is grateful to the ESO C\&EE and the director of the OHP for financial support and hospitality. VEK and JV gratefully acknowledge the financial support and hospitality of the Astrophysical Institute Potsdam during several visits. TXT is grateful for the hospitality of Chantal Balkowski at the Observatoire de Meudon and of Laurent Vigroux at the Service d'Astrophysique at Saclay. He thanks the partial financial support of NASA grant 5-2060.

\section{References}

Bender R., Möllenhoff C., 1987, A\&A 177, 71

Binggeli B., Sandage A., Tammann G.A., 1988, ARA\&A 26, 509

Caon N., Capaccioli M., D’Onofrio M., 1993, MNRAS 265, 1013

Côté S., Freeman K., Carignan C., 1994, "Dwarf Galaxies". In: Meylan G., Prugniel P. (eds.), ESO Conference and Workshop Proc. 49, 101

de Vaucouleurs G., 1948, Ann. Astrophys. 11, 247 
de Vaucouleurs G., de Vaucouleurs A., Corwin H.G., Buta R.J., Paturel G., Fouqué P., 1991, Third Reference Catalogue of Bright Galaxies, I-III. Springer-Verlag

Fisher J.R., Tully R.B., 1975, A\&A 44, 151

Fisher J.R., Tully R.B., 1981, ApJS 47, 139

Huchtmeier W.K., Skillman E.D., 1994, "Dwarf Galaxies". In: Meylan G., Prugniel P. (eds.), ESO Conference and Workshop Proc. 49, 299

Karachentseva V.E., Sharina M.E., 1988, Comm. Spec. Astroph. Obs. 57 (KS)

Karachentseva V.E., 1990, Pis'ma Astron. Zh. 16, 99

Karachentseva V.E., Vavilova I.B., 1994a, Bull. Spec. Astr. Obs. 37, 92

Karachentseva V.E., Vavilova I.B., 1994b, "Dwarf Galaxies". In: Meylan G., Prugniel P. (eds.), ESO Conference and Workshop Proc. 49, 91

Karachentseva V.E., Vavilova I.B., 1995, Kinematika i fizika nebesnych tel, 11, 46

Karachentsev I.D., Tikhonov N.A., Sazonova L.N., 1994, Lett. Soviet Astron. Zh. 20, 90

Landolt A.U., 1992, AJ 104, 340

Lorenz H., Richter G.M., Capaccioli M., Longo G., 1993, A\&A 277,321

Mazzarella J.M., Balzano V.A., 1986, ApJS 62, 751

Nilson P., 1973, Uppsala General Catalogue of Galaxies,
Uppsala Obs. Ann. 6 (UGC)

Paturel G., Fouqué P., Bottinelli L., Gouguenheim L., 1989, Catalogue of Principal Galaxies, Lyon (PGC)

Reaves G., 1983, "Star-forming dwarf galaxies and related objects". In: Knuth D., Thuan T.-X., Tran Thanh Van J. (eds.). Edition Frontiers

Rönnback J., Bergvall N., 1994, A\&A 108, 193

Sandage A., Binggeli B., Tammann G.A., 1985, AJ 90, 1759

Schneider S.E., Thuan T.X., Magri C., Wadiak J.E., 1990, ApJS 72, 245 (STMW)

Schneider S.E., Thuan T.X., Mangun J.G., Muller J., 1992, ApJS 81, 5 (STMM)

Sersic J.-L., 1968, Atlas de galaxias australes, Observatorio Astronomico, Cordoba

Staveley-Smith L., Davies R.D., Kinman T.D., 1992, MNRAS 258, 334 (SDK)

Thuan T.X., Seitzer P., 1979, ApJ 231, 327 (TS)

Tikhonov N.A., Sazonova L.N., 1994, A\&AS (in press)

Vader J.P., Chaboyer B., 1994, AJ 108, 1209

Vennik J., Richter G.M., 1994, Astron. Nachr. 315, H3, 245

Zaritsky D., 1994, "Dwarf Galaxies". In: Meylan G., Prugniel P. (eds.), ESO Conference and Workshop Proc. 49, 361

Zickgraf F.-J., Humphreys R.M., Sitko M.L., Manley T., 1990, PASP 102, 925 

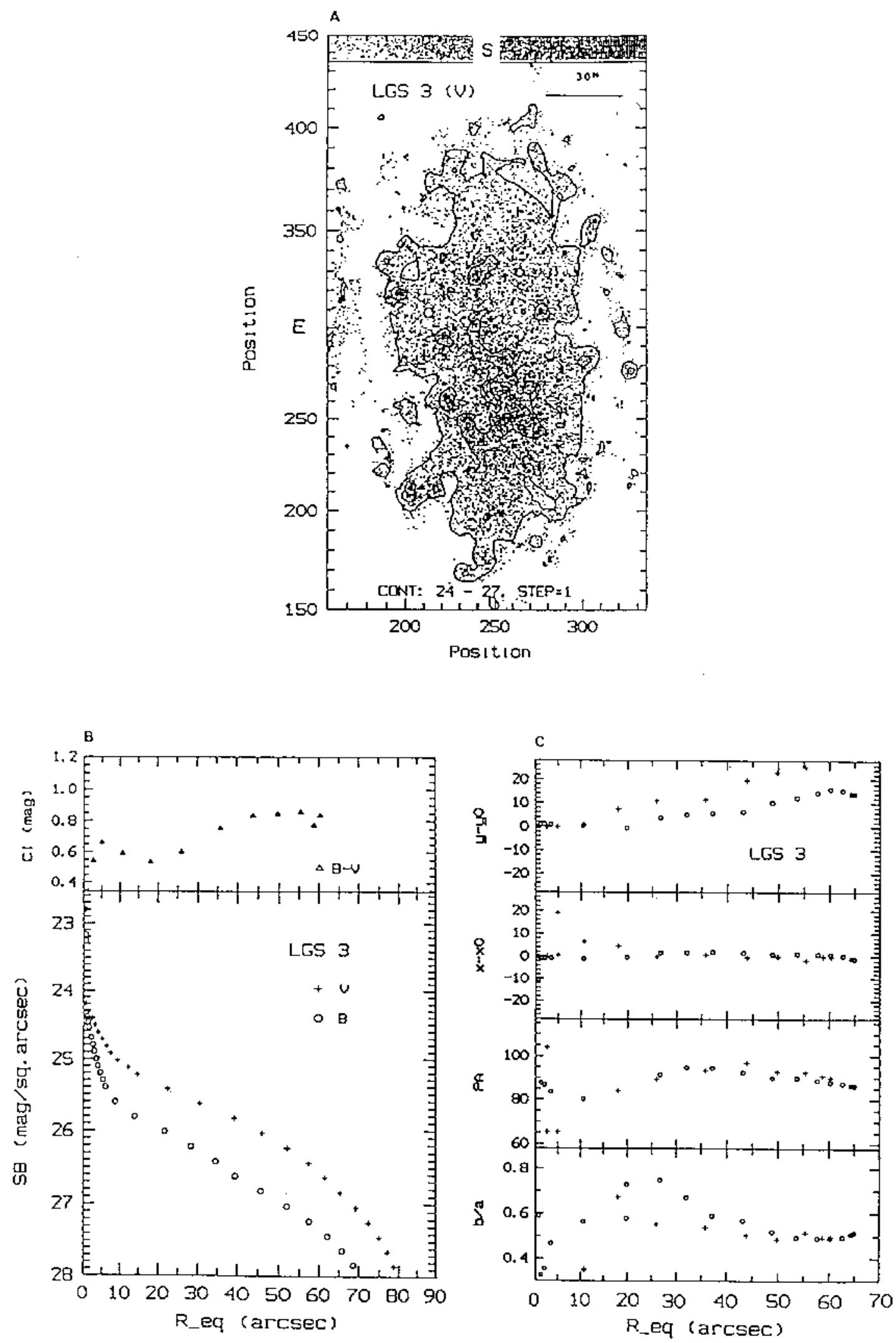

Fig. 3. -18. Panel A: Grey scale representation of the galaxy frame with orientation and scale bar. Position is given in pixels with a scale $0.75^{\prime \prime} /$ pixel. North is at bottom, East is to the left. Several isophotes are plotted starting from the $26.5 \mathrm{mag} / \square^{\prime \prime}$, as the lowest surface brightness level and with a step of $0.5 \mathrm{mag} / \square^{\prime \prime}$, if not indicated otherwise. Panel B: The equivalent $B$ (circles) and $V$ (crosses) surface brightness profiles, derived by means of the Potsdam surface photometry routines, and the colour index (triangles) profile are plotted as a function of equivalent radius. Panel C: Radial profiles of parameters of fitted ellipses, which include the axis ratio $(b / a)$, major axis position angle (PA, measured counterclockwise from the positive $x$-axis on the observed frames as shown in panel A) and displacements of the center of fitted ellipses both in $x\left(x-x_{0}\right)$ and in $y\left(y-y_{0}\right)$ in arcseconds, where $\left(x_{0}, y_{0}\right)$ is the center of the innermost ellipse. Circles refer to the $B$ frame, crosses - to the $V$ frame 

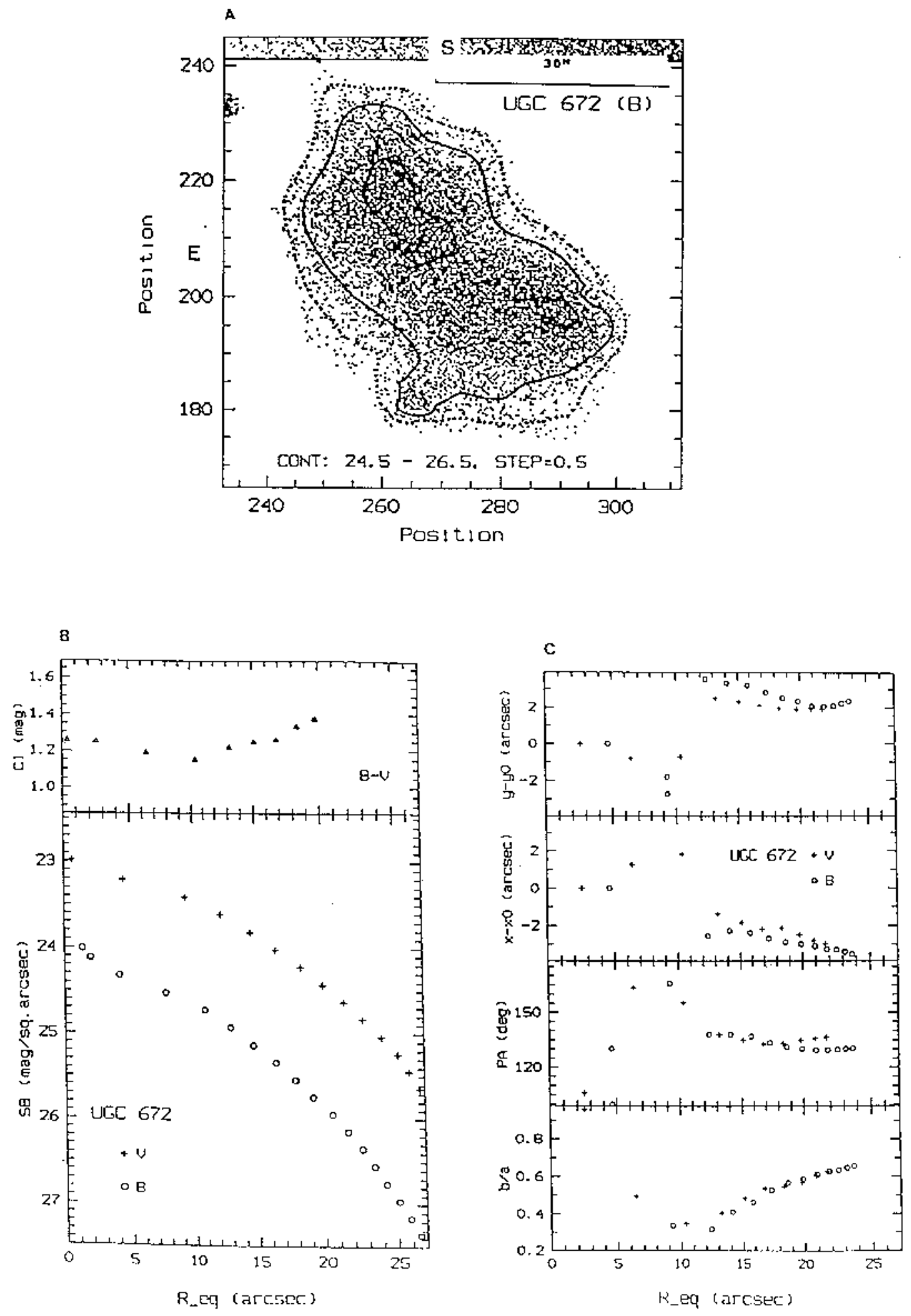

Fig. 4. 

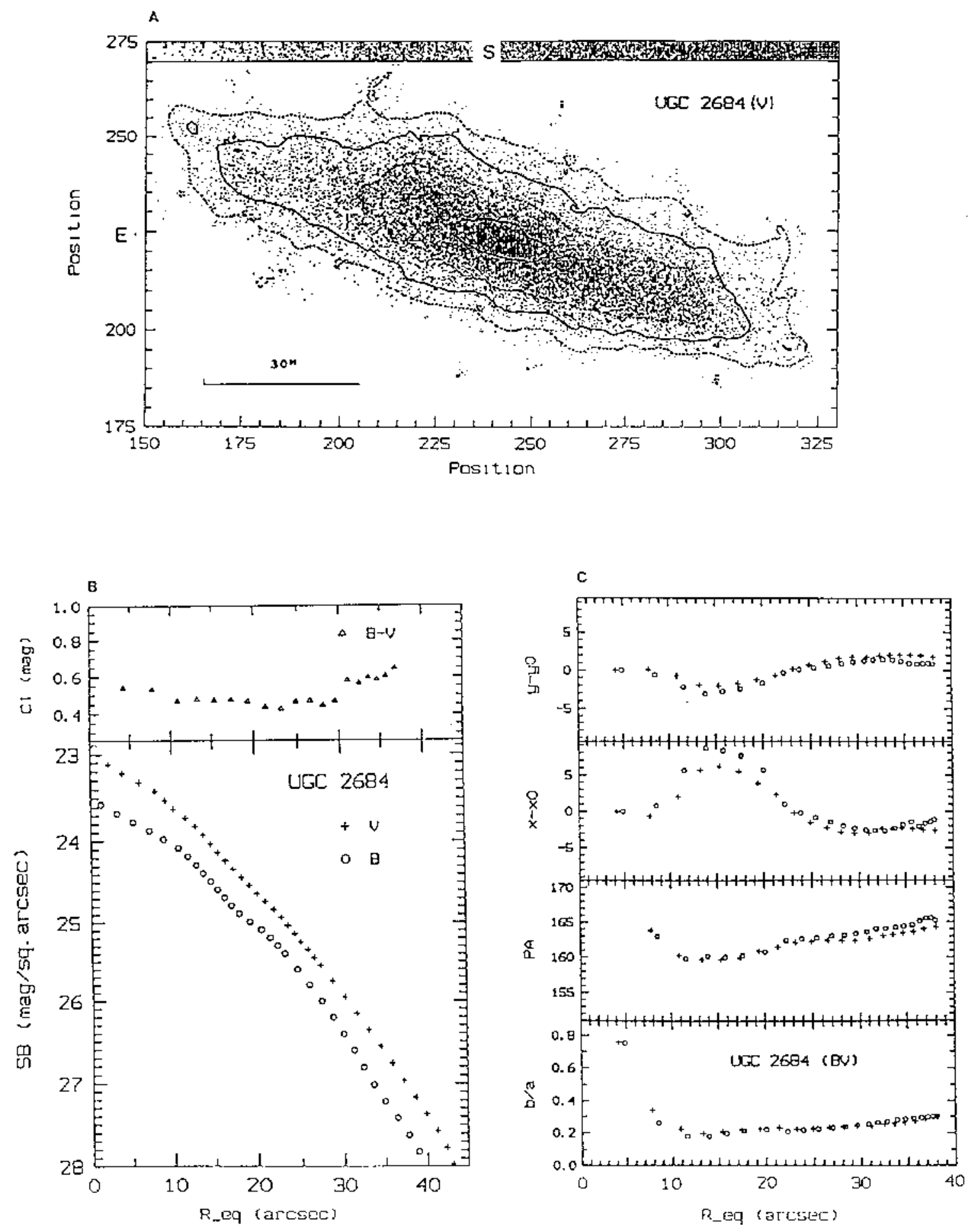

Fig. 5. 

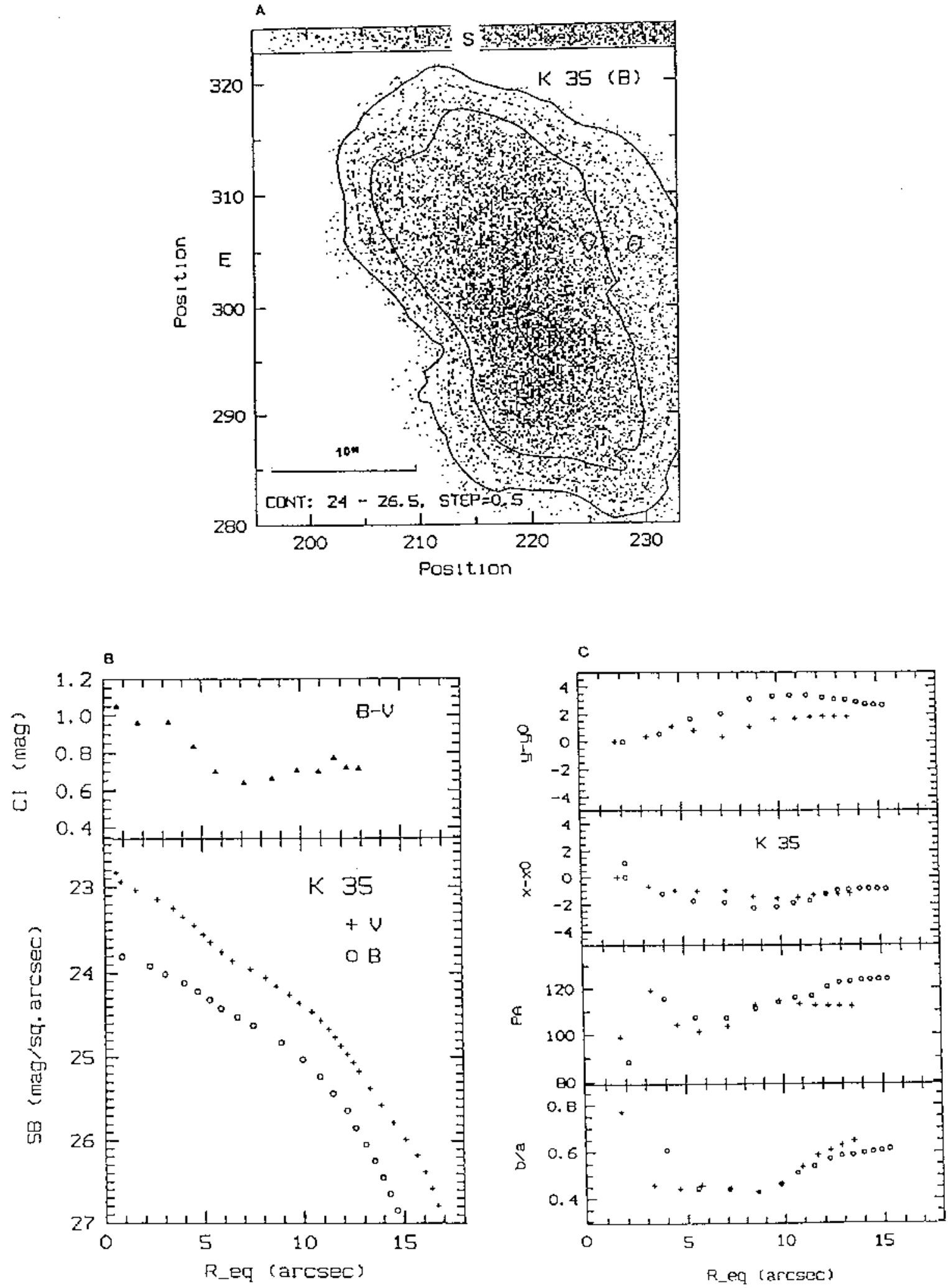

Fig. 6. 

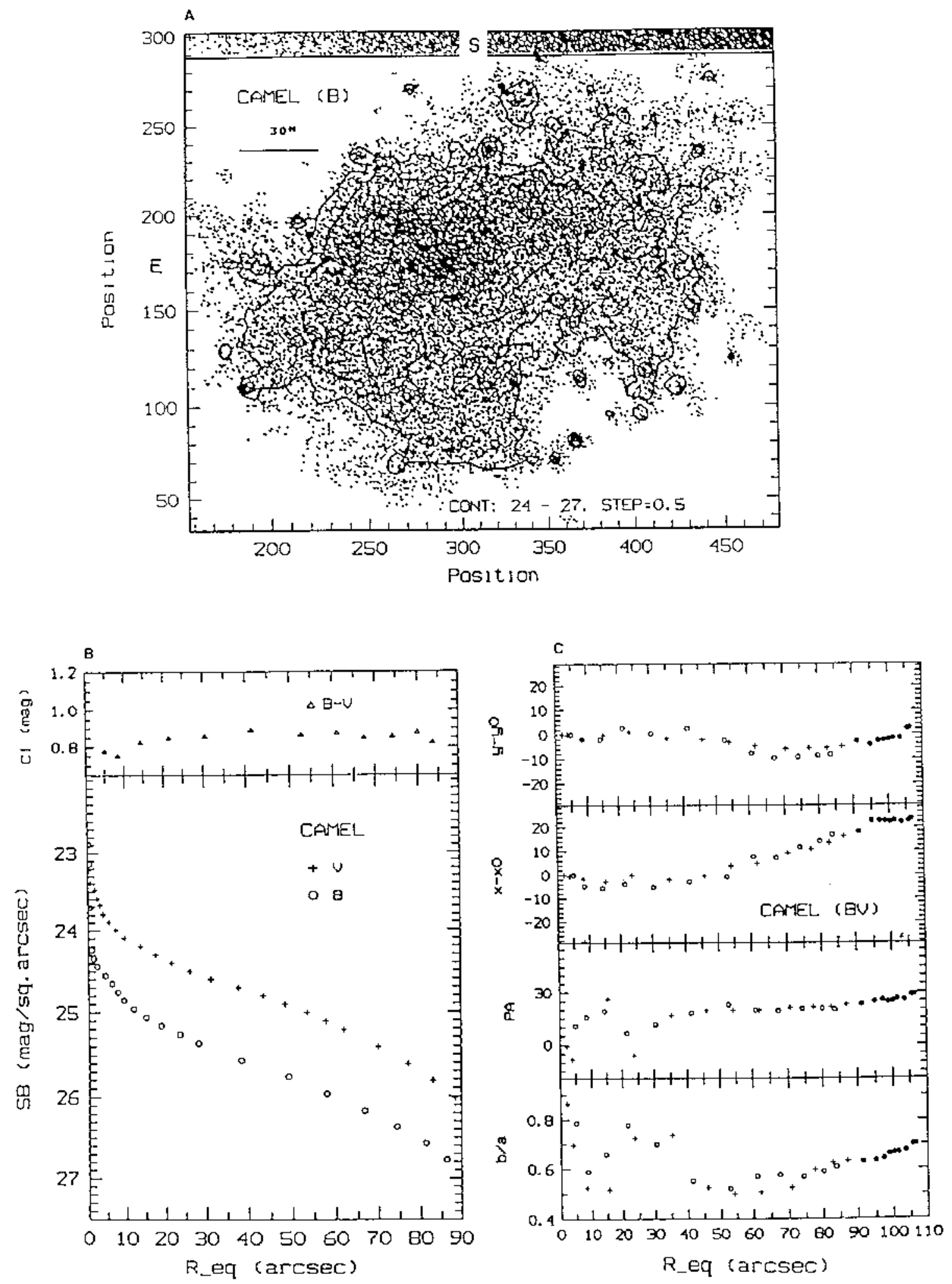

Fig. 7. 

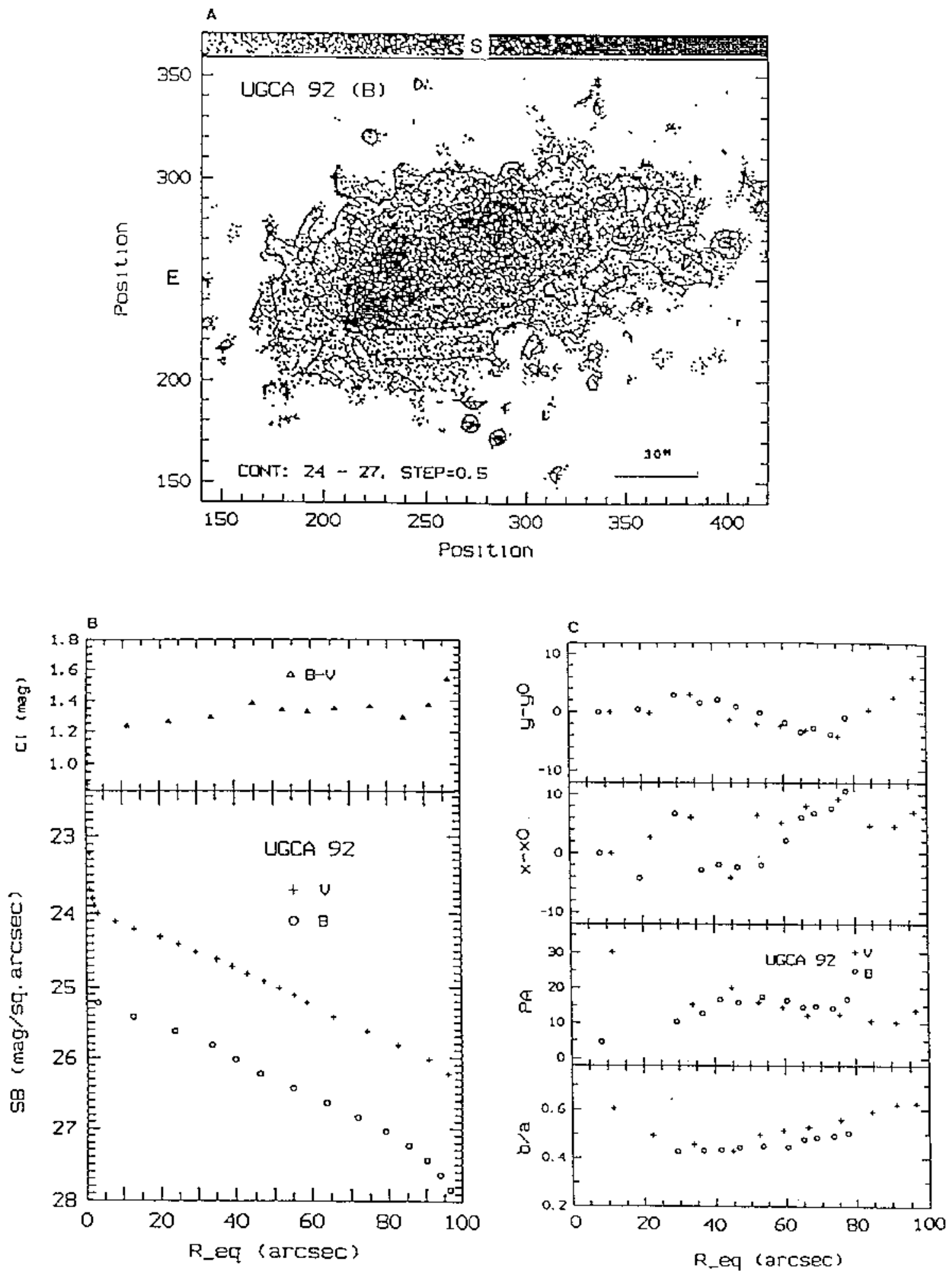

Fig. 8. 

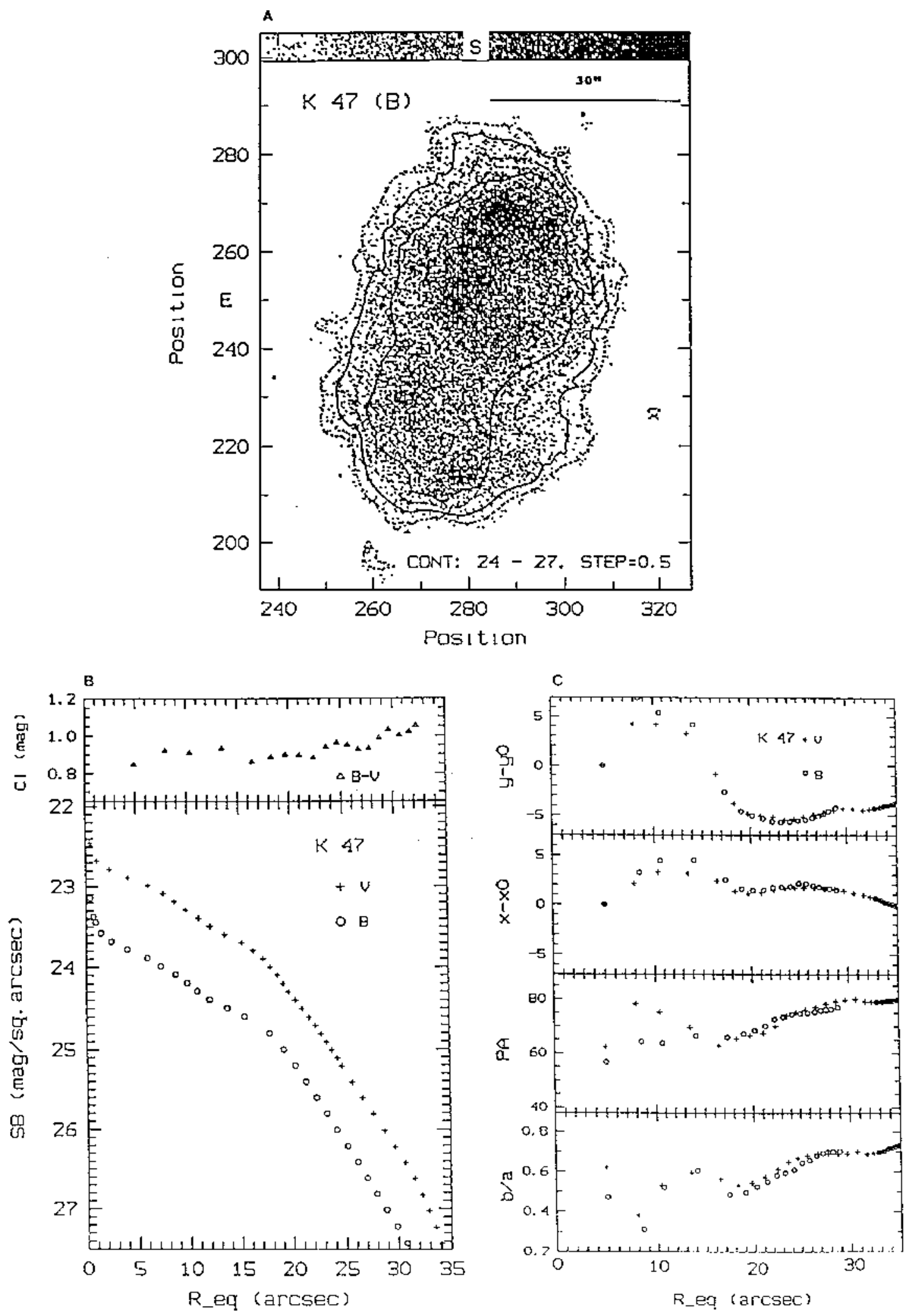

Fig. 9. 

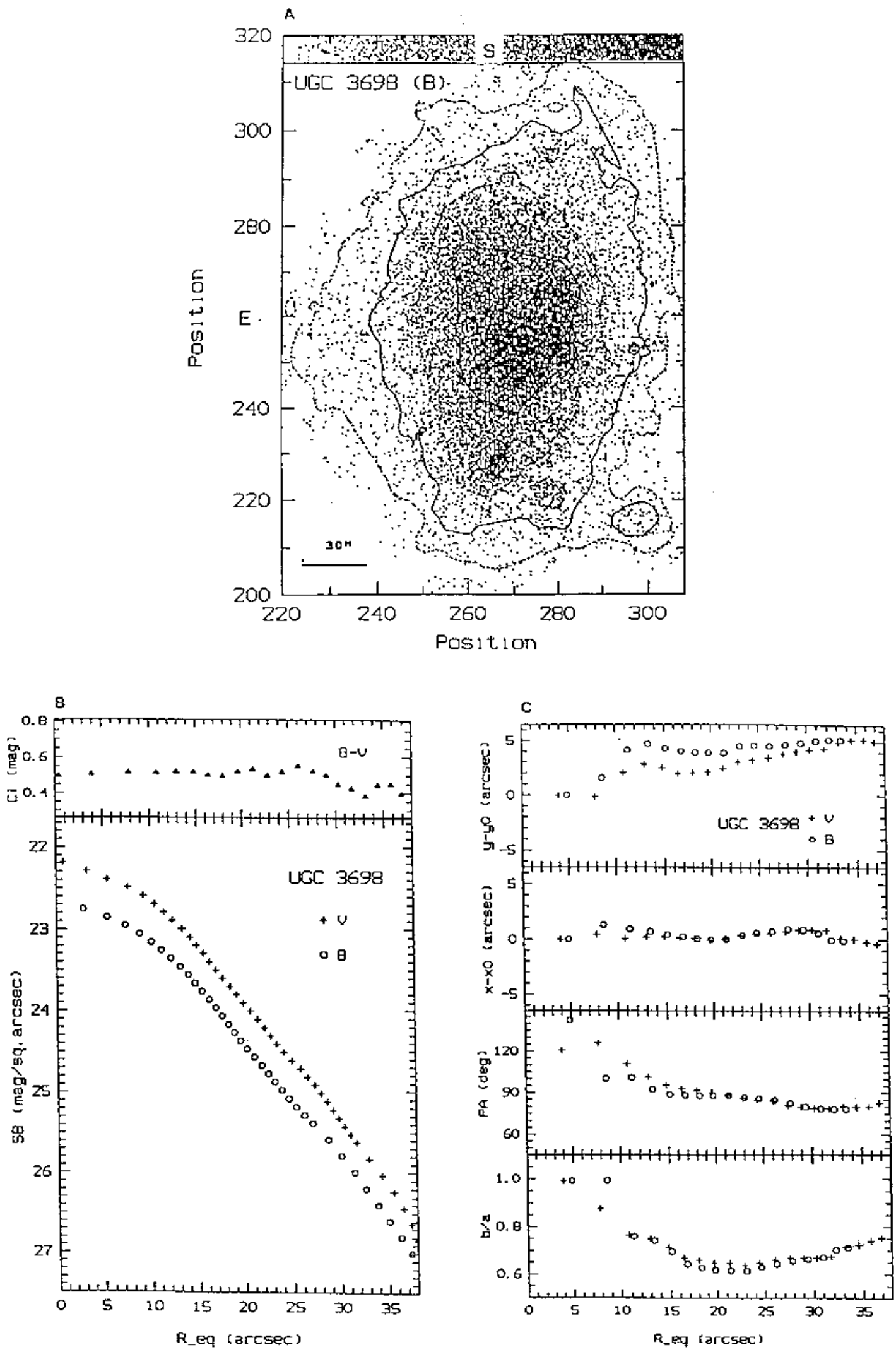

Fig. 10. 

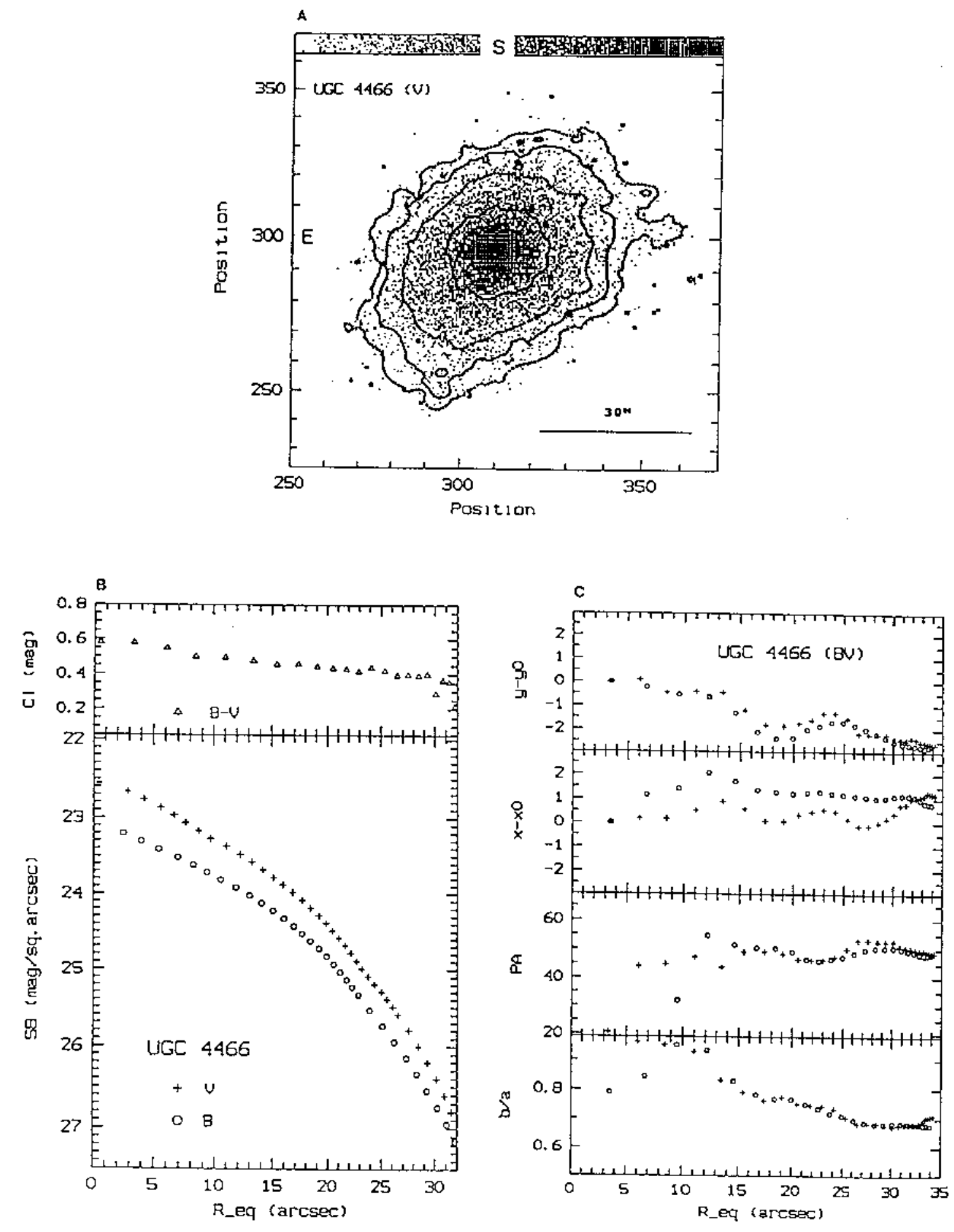

Fig. 11 . 

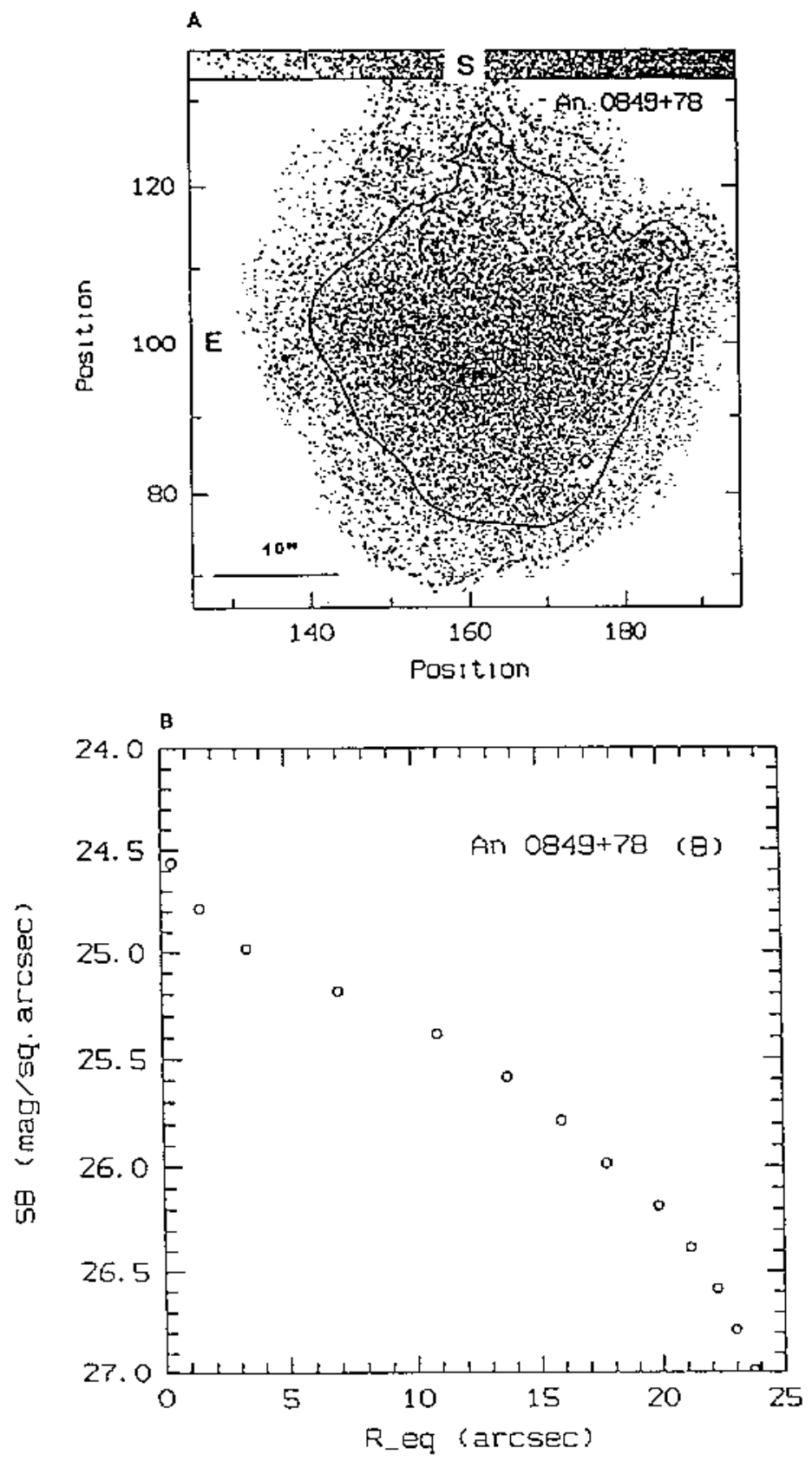

Fig. 12. 

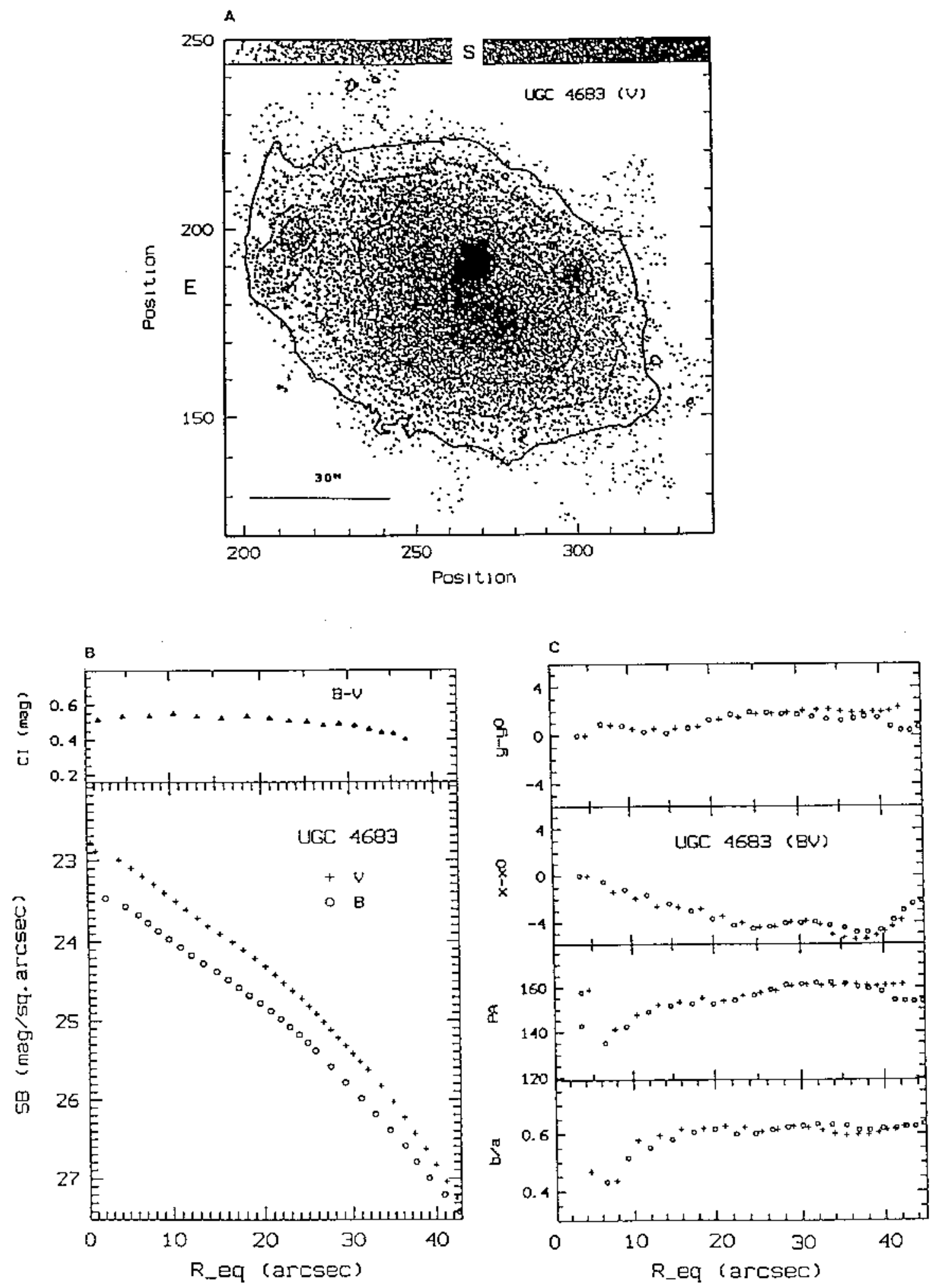

Fig. 13. 

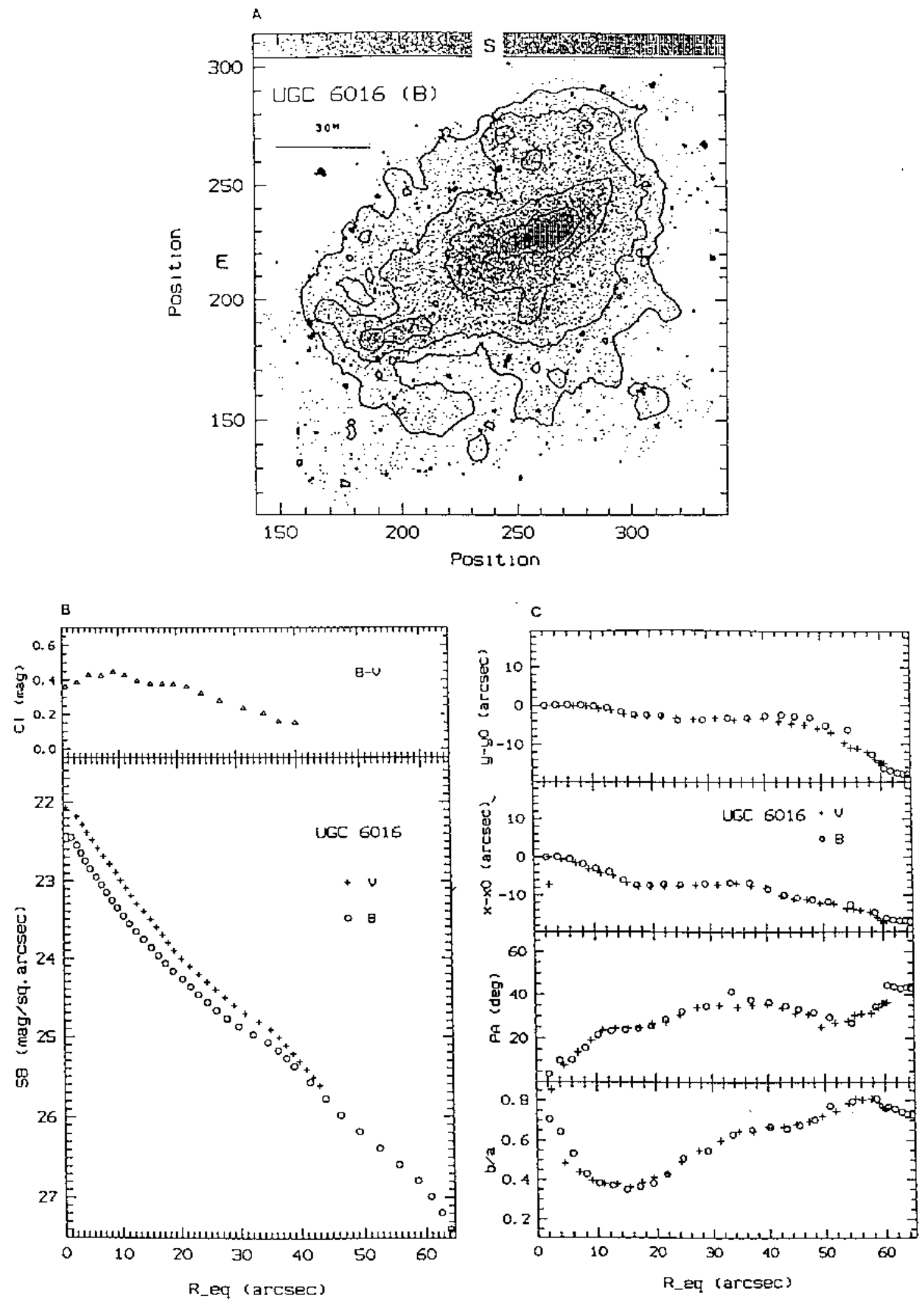

Fig. 14 . 

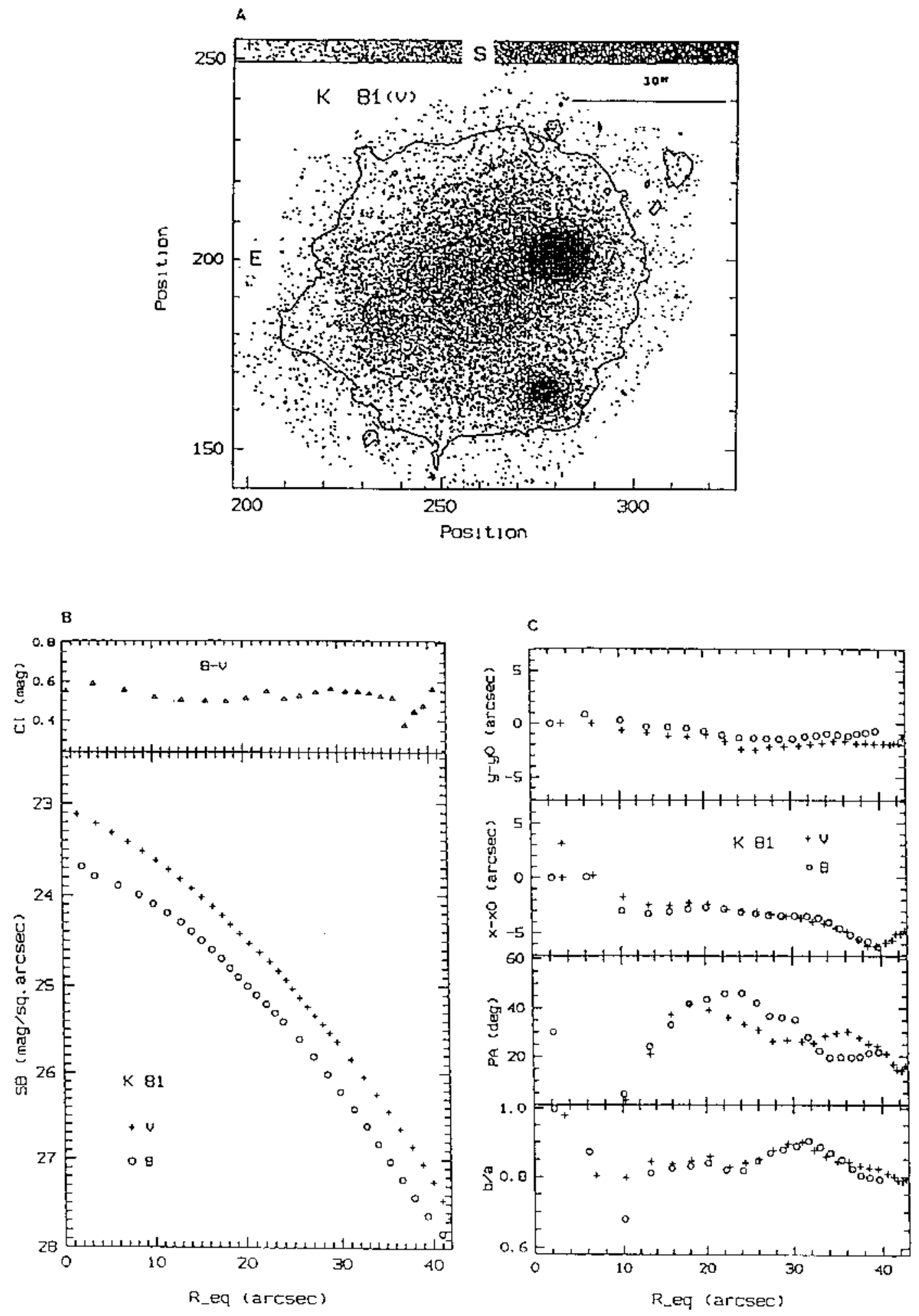

Fig. 15. 

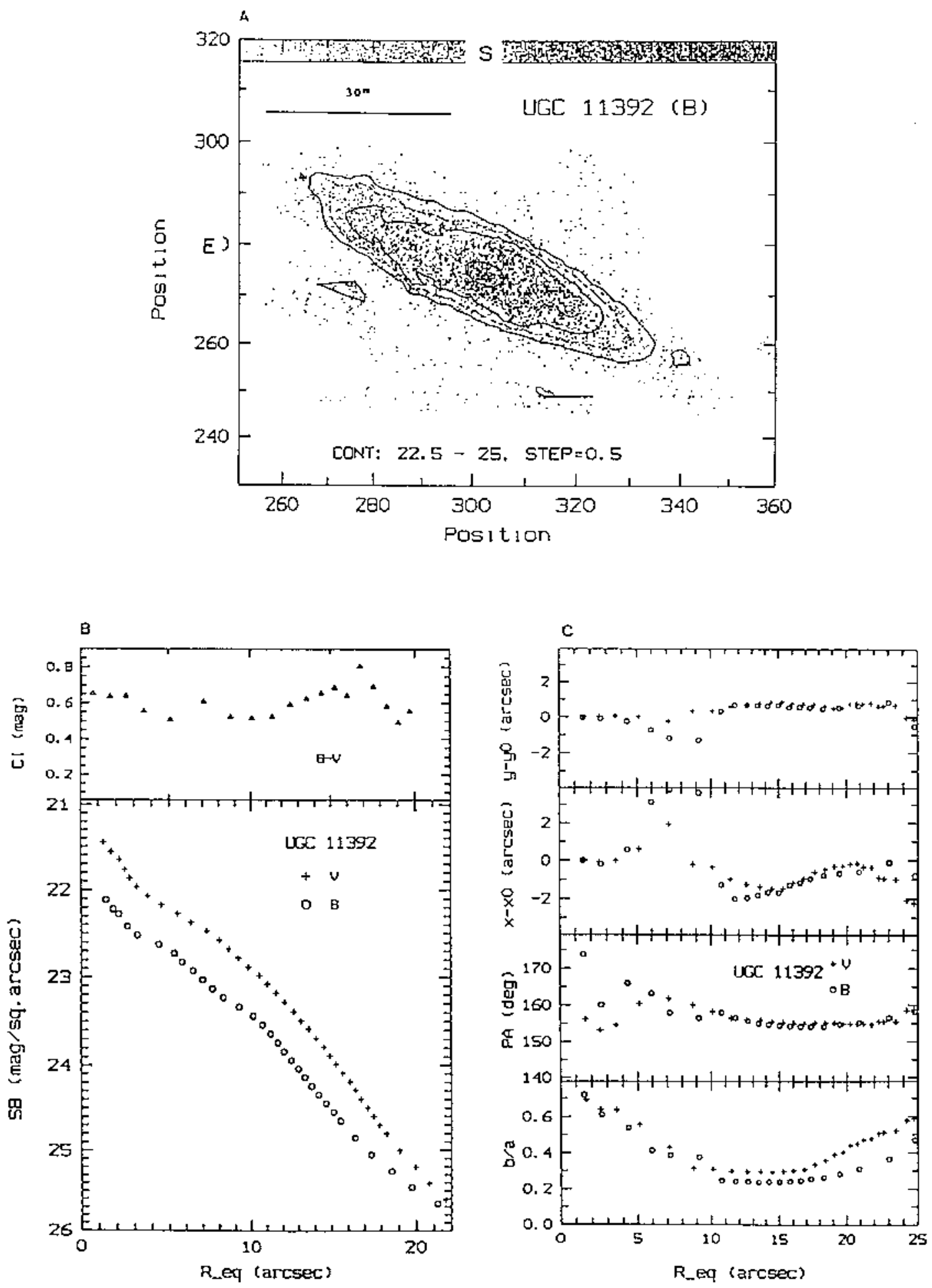

Fig. 16. 

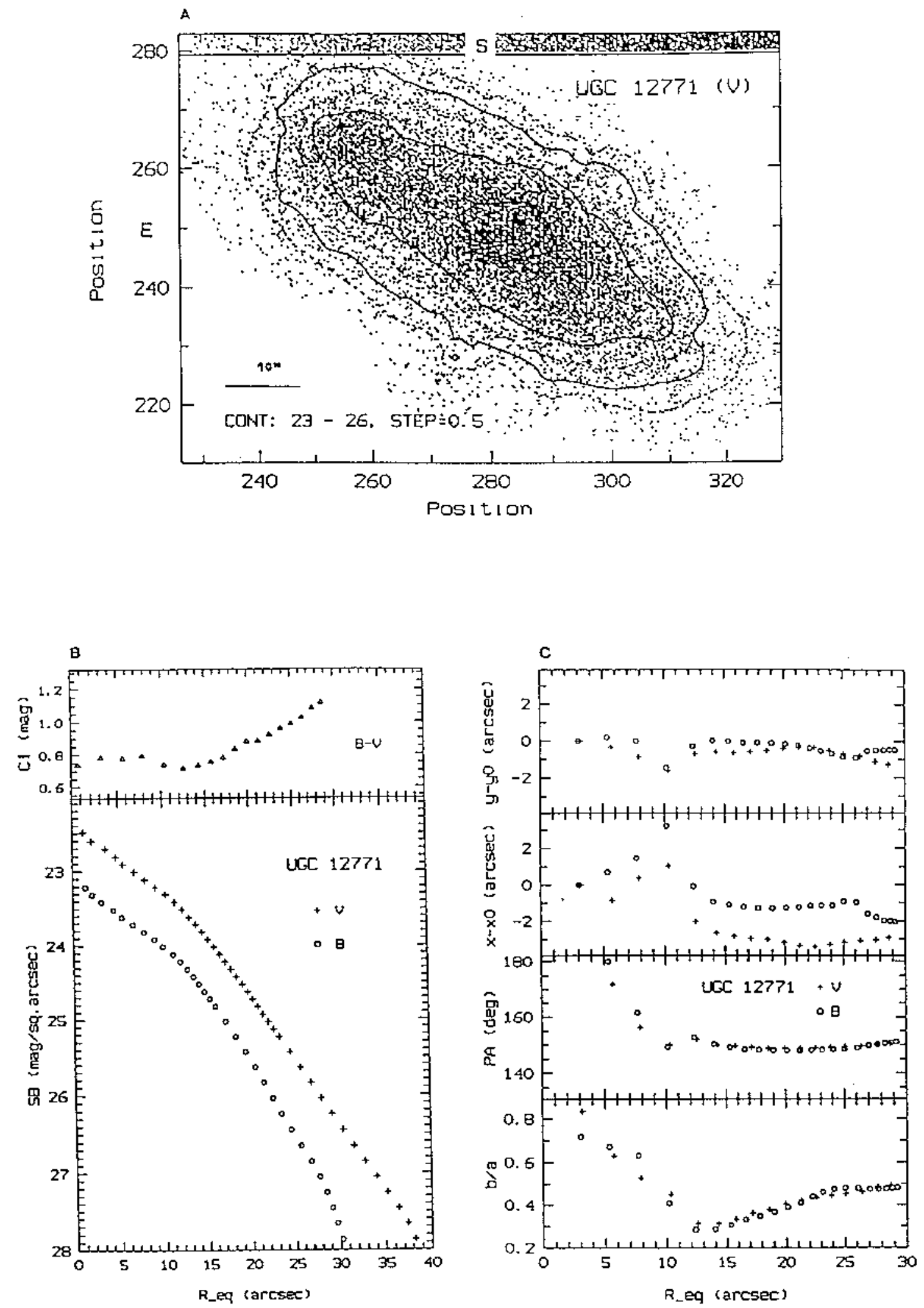

Fig. 17. 

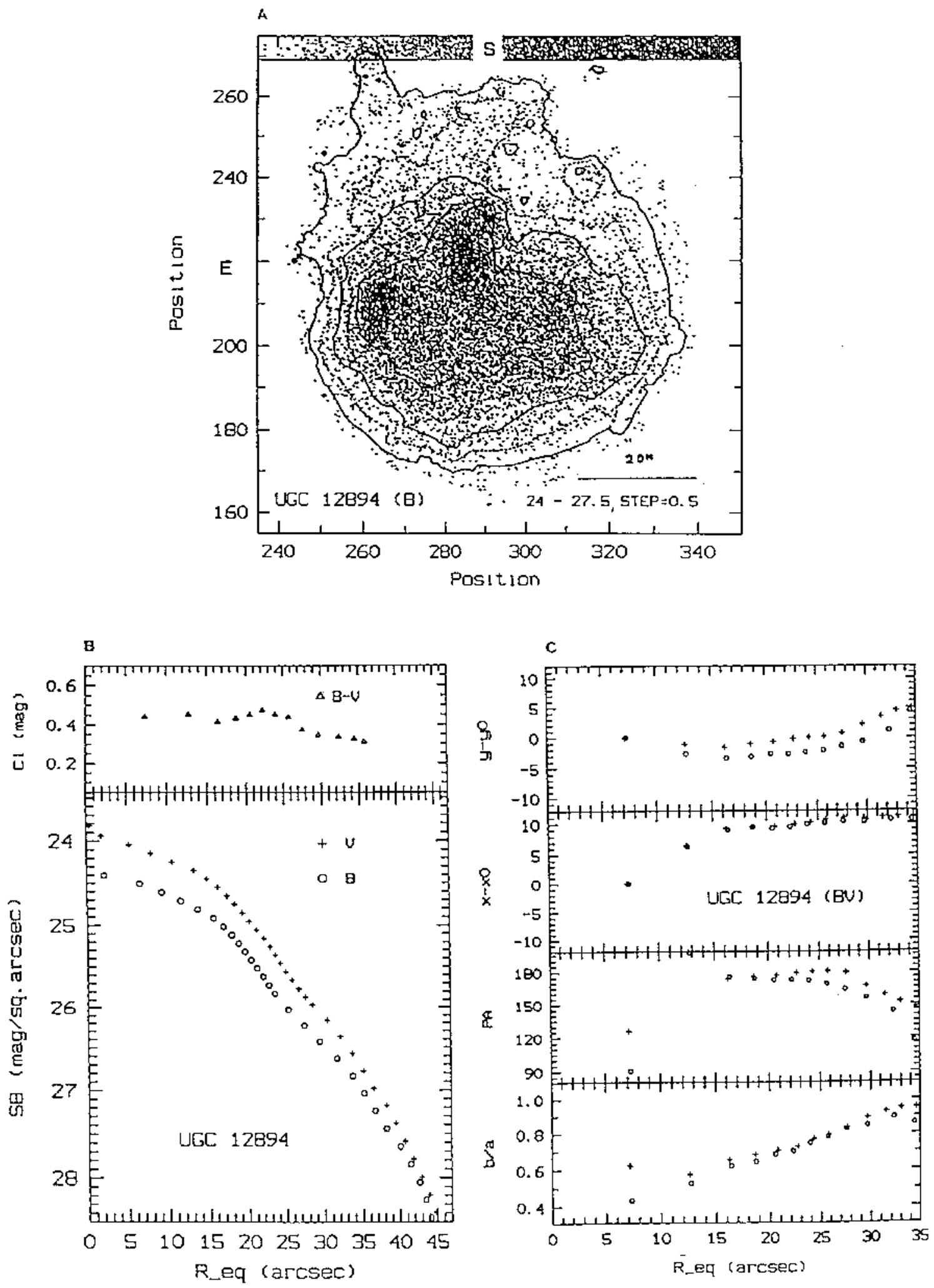

Fig. 18. 